\title{
Tandem-L: A Technical Perspective on Future Spaceborne SAR Sensors for Earth Observation
}

\author{
Sigurd Huber, Felipe Queiroz de Almeida, Michelangelo Villano, Member, IEEE, Marwan Younis, Senior \\ Member, IEEE, Gerhard Krieger, Fellow, IEEE, Alberto Moreira, Fellow, IEEE
}

\begin{abstract}
Tandem-L is proposed as a spaceborne SAR mission developed and operated by the German Aerospace Center in cooperation with several Helmholtz research centers and the German space industries. The mission concept comprises two fully polarimetric radar satellites providing mono- and bi-static SAR imagery. A key feature of these SAR sensors is the employment of large light-weight unfurlable mesh reflectors fed by digital feed arrays. The main advantage of this new SAR system concept is the provision of large antenna apertures in space and flexible operation via reconfigurable feed electronics. By this, it becomes possible to map, for the first time, a continuous $350 \mathrm{~km}$ wide swath with $7 \mathrm{~m}$ azimuth resolution with excellent noise equivalent sigma zero (NESZ) and ambiguity suppression.
\end{abstract}

This paper shall give an overview on the technical aspects of the Tandem-L SAR instrument and antenna design. In particular, after a short review of the SAR system requirements, the concept of reflector SAR systems is outlined and the operation principle is presented. General guidelines for the design of array-fed reflector antennas with application to SAR imaging are given. Then the optimization approach of the feed array design is detailed, with specific emphasis on a fixed beamforming concept in azimuth. In this context also the problem of cross-pol pattern mitigation is addressed. These optimization steps are shown to be crucial for achieving the performance requirements in quadpol acquisitions. Beamforming in elevation is performed on board the spacecraft via digital hardware. This paper presents the beamforming architecture on receive for Tandem-L, which would apply in general for instance also to planar multi-elevation beam SAR antennas with ScanOn-Receive capabilities. Tandem- $L$ is operated as a staggered SAR, which means varying the pulse repetition interval from pulse to pulse. In this context the major design challenges are presented. Moreover, the impact of pulse staggering on the imaging performance is discussed. Tandem-L's SAR performance is presented by means of numerical simulations showing that the performance requirements imposed by the scientific user community could be met. The final part of the paper addresses options for high azimuth resolution imaging as well as a beamforming method for enhanced range ambiguity suppression.

Index Terms-synthetic aperture radar (SAR), spaceborne SAR mission, array-fed reflector, high-resolution wide-swath (HRWS), multichannel system, digital beamforming (DBF)

\section{INTRODUCTION}

$\mathbf{T}$ ANDEM-L is a fully polarimetric bistatic spaceborne L-band SAR mission with the goal of monitoring dynamic processes on Earth's surface [1]-[7].

Manuscript received March 4, 2019. S. Huber, F. Queiroz de Almeida, M. Villano, M. Younis, G. Krieger and A. Moreira are with the Microwaves and Radar Institute, German Aerospace Center (DLR), Oberpfaffenhofen, Germany, e-mail: sigurd.huber@dlr.de.
Important applications are regular inventories of globalscale biomass [8], systematic measurements of surface deformation with millimeter accuracy, the generation of digital surface and terrain models, as well as disaster monitoring related to flooding events, earthquakes and volcano eruptions.

The Tandem-L radar satellites make use of large unfoldable mesh reflector antennas, which have been identified as ideal for highly sensitive, high-resolution wideswath (HRWS) SAR acquisitions [9]. Figure 1 presents an artist's conception of Tandem-L. In this context a multi-

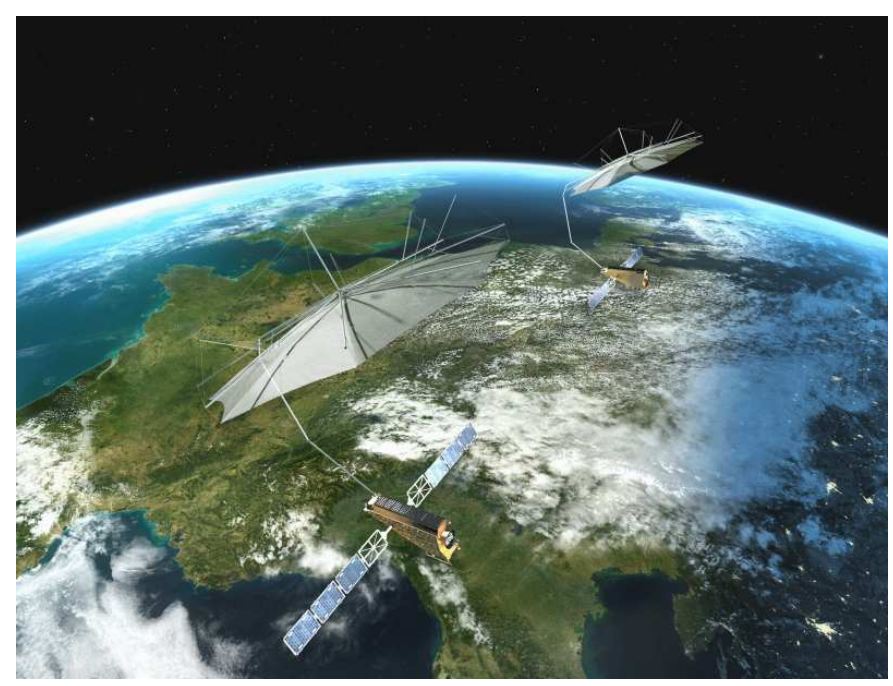

Figure 1: Concept art graphic showing the Tandem-L SAR satellites in orbit over Europe.

channel digital feed array architecture will be employed, whereby the radar echoes received by the individual feed elements are digitized and processed on board the spacecraft. This concept offers the potential to realize gapless SAR imaging over a large swath width with high azimuth resolution.

The Tandem-L SAR imaging concept requires the gapless acquisition of swathes with a width of up to $350 \mathrm{~km}$ and $7 \mathrm{~m}$ azimuth resolution. These demanding requirements have a significant impact on system design and have led to the development of advanced radar signal processing techniques. This paper details some of the design and radar signal processing concepts to be implemented on Tandem-L. The paper is organized as follows: Section II gives an overview of the main SAR system requirements. In section III, the SAR antenna and 
instrument architecture are introduced. Section IV details operational and processing related concepts. Section $\mathrm{V}$ presents the SAR imaging performance for the main Tandem-L modes. In section VI options for high azimuth resolution imaging and potentials for performance improvement are discussed.

\section{TANDEM-L SAR System Requirements}

The Global Climate Observing System (GCOS) has identified a list of so-called Essential Climate Variables (ECVs) in the atmospheric, ocean and terrestrial domains. The monitoring and quantification of these ECVs is highly important in the context of the United Nation Framework Convention on Climate Change (UNFCCC) and Intergovernmental Panel on Climate Change (IPCC) [10]. The Tandem-L mission is an opportunity to provide to the scientific community data related to the ECVs at an unprecedented scale.

The Tandem-L science team comprises approximately 180 national and international members, with the Helmholtz Alliance as core of this research framework. The scientific mission requirements and products were iterated and refined in cooperation with further partners and in further research programmes (e.g. network EOS, the geotechnology programme Exupery, TERENO and ACROSS) [11]. In this sense the Tandem-L performance specifications represent a compromise in the scientific user community with regard to the data quality to be expected from this mission.

Tandem-L shall be operated in a $740 \mathrm{~km}$ orbit with a 16-day repeat cycle. The two spacecraft are supposed to acquire SAR data over a mission lifetime of 1012 years. Depending on the mode, signal bandwidths

\begin{tabular}{l|c|c} 
polarization & single/dual & quad \\
\hline swath width & $350 \mathrm{~km}$ & $175 \mathrm{~km}$ \\
max. signal bandwidth & $84 \mathrm{MHz}$ & $84 \mathrm{MHz}$ \\
gr. range res. (near/far) & $4.0 \mathrm{~m} / 2.4 \mathrm{~m}$ & $3.8 \mathrm{~m} / 2.8 \mathrm{~m}$ \\
min. azimuth resolution & $7 \mathrm{~m}$ & $7 \mathrm{~m}$ \\
NESZ $(20+5 \mathrm{MHz})$ & $<-30 \mathrm{~dB}$ & $<-32 \mathrm{~dB}$ \\
NESZ $(40+5 \mathrm{MHz})$ & $<-28 \mathrm{~dB}$ & $<-30 \mathrm{~dB}$ \\
NESZ $(84 \mathrm{MHz})$ & $<-25 \mathrm{~dB}$ & $<-28 \mathrm{~dB}$ \\
$A S R$ & $<-25 \mathrm{~dB}$ & $<-25 \mathrm{~dB}($ co-pol) \\
ASR & - & $<-22 \mathrm{~dB}(\mathrm{cx}-$ pol)
\end{tabular}

Table I: Main Tandem-L imaging mode requirements. The total ambiguity-to-signal ratio $A S R$ accounts for range and azimuth ambiguities. For the quad polarization mode the $A S R$ requirement for the cross polarization channels has been relaxed to $<-22 \mathrm{~dB}$.

ranging from $20 \mathrm{MHz}$ to $84 \mathrm{MHz}$ within L-band between $1.215 \mathrm{GHz}$ and $1.3 \mathrm{GHz}$ shall be employed. This corresponds to $7.5 \mathrm{~m}$ to $1.8 \mathrm{~m}$ slant range resolution, while an azimuth resolution of $7 \mathrm{~m}$ is being considered as baseline. Table I lists the main Tandem-L imaging modes. Single and dual polarization data are acquired over a $350 \mathrm{~km}$ swath, while the quad polarization mode covers a swath in the order of $175 \mathrm{~km}$. The requirements are specified via the noise equivalent sigma zero (NESZ) and the range and azimuth ambiguity-to-signal ratio ${ }^{1}$ $(A S R)$. Depending on the application, Tandem-L features several other observation modes, with reduced range resolution. For example the $20+5 \mathrm{MHz}$ split-band mode will have a better sensitivity compared to the $84 \mathrm{MHz}$ mode such that NESZ values below $-30 \mathrm{~dB}$ can be expected.

\section{TANDEM-L SAR System Concept}

The operation principle of an array-fed reflector SAR system is illustrated in Fig. 2 [12]-[16]. The reflector is illuminated by an array of feed elements, which are typically patch antennas at L-band. On transmit, all elements are activated simultaneously such that the entire swath is covered by a wide antenna footprint. In contrast to direct radiating arrays, in the reflector case each feed element illuminates a distinct spot in this footprint. This means, there is a direct mapping between the feed array topology and the position on the Earth surface. On receive, typically two to seven elements are used in each elevation column to track the radar echo from the ground, employing digital beamforming (DBF) techniques [17]-[20]. The number of active elevation elements depends on the feed element spacing. After elevation beamforming, the associated signals are then downlinked to the ground for further processing. For reasons of hardware complexity and data rate constraints Tandem-L will employ a combination of azimuth feed elements forming a single channel to be downlinked to ground. Insofar, with regard to the azimuth channels, the Tandem- $\mathrm{L}$ feed array could be regarded as one-dimensional. This Tandem-L feed array concept is explained in detail in the next section.

\section{A. Feed-Array Design}

To design and evaluate the performance of this coupled electromechanical feed-reflector system, it is necessary to consider near-field electromagnetic interactions. The approach adopted for Tandem-L is to simulate plane waves originating from the antenna footprint on ground. These plane waves will generate an electromagnetic field in the domain of the focal plane. The corresponding feed elements would then be placed at the maximum of the field collecting a certain percentage of the field power. The response of the entire feed array results from the superposition of all incident plane waves originating from within the antenna footprint. Figures $3 \mathrm{a}$ to $3 \mathrm{c}$ show examples of field distributions in the feed array plane. The central beam near the focus of the reflector shows a compact energy distribution (Fig. 3b). The outer beams exhibit defocusing (Figs. 3a,c). The coverage with feed patch elements for a given spacing in elevation and azimuth can be found via a power constraint

$$
\sum_{i} \iint_{A_{\mathrm{p}, i}}\left|\operatorname{Re}\left\{S_{z}(x, y)\right\}\right| \mathrm{d} x \mathrm{~d} y \geq \eta P_{\text {tot }},
$$

${ }^{1}$ The range and azimuth ambiguity-to-signal ratio approximately equals the sum of the range ambiguity-to-signal ratio (RASR) and the azimuth ambiguity-to-signal ratio $(A A S R)$ 


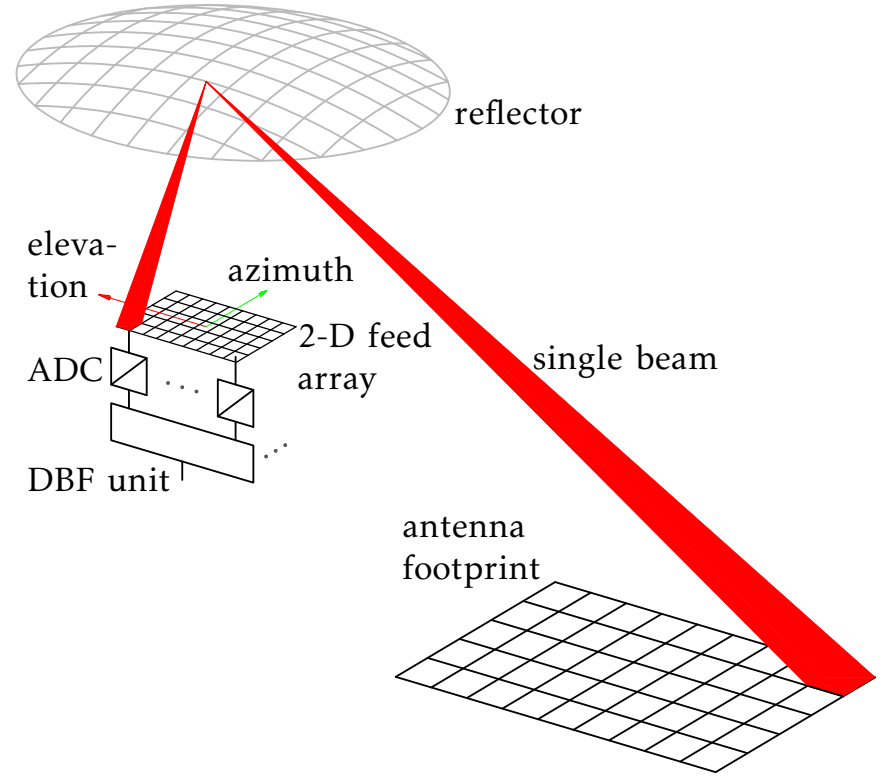

Figure 2: Array-fed reflector principle. Each cell in the antenna footprint is associated with an element in the 2-D feed array. On transmit a wide beam is generated by simultaneously activating all feed elements. On receive a high gain beam follows the pulse echo on ground.

where $S_{z}$ is the $z$-component of the Poynting vector in the feed plane. This inequality states that the power propagating through the electrical area of the $i$ th patch element $A_{\mathrm{p}, i}$ is added until a certain percentage $\eta(80 \%$ in Fig. 3) of the power of the corresponding incident plane wave $P_{\text {tot }}{ }^{2}$ is collected. Important to mention is that the sum in equation (1) is ranked, starting with the patch element catching the highest power. Combining all patches yields the transmit beam pattern. The 'boneshaped' flair of the energy distribution away from the center is a consequence of the defocusing. The Tandem$\mathrm{L}$ feed array has a length of $5.5 \mathrm{~m}$ and a width of $0.86 \mathrm{~m}$ (see Table II). The choice for this particular feed array length is mainly driven by the need of accommodating the two spacecraft in a single launcher. Commonly, the element spacing is taken larger than $0.6 \lambda$, ensuring that coupling effects can be neglected. In principle coupling is not an issue, as long as the coupling matrix is known. In general, SAR antenna models are based on so called embedded patterns, which take into account electric interactions of the radiating element with the surrounding antenna structure. In case of Tandem-L, additional considerations, like $\mathrm{T} / \mathrm{R}$-module redundancy and a specific tile structure of the feed array, led to the particular number of 35 elevation elements with a spacing of $0.6591 \lambda$. In azimuth, the field distribution could be covered by six elements spaced $0.6 \lambda$. Here, the choice of an even number was motivated by a cross polar cancellation technique, where always two neighboring

\footnotetext{
${ }^{2} P_{\text {tot }}$ is the power incident on the projected aperture area of the reflector.
}
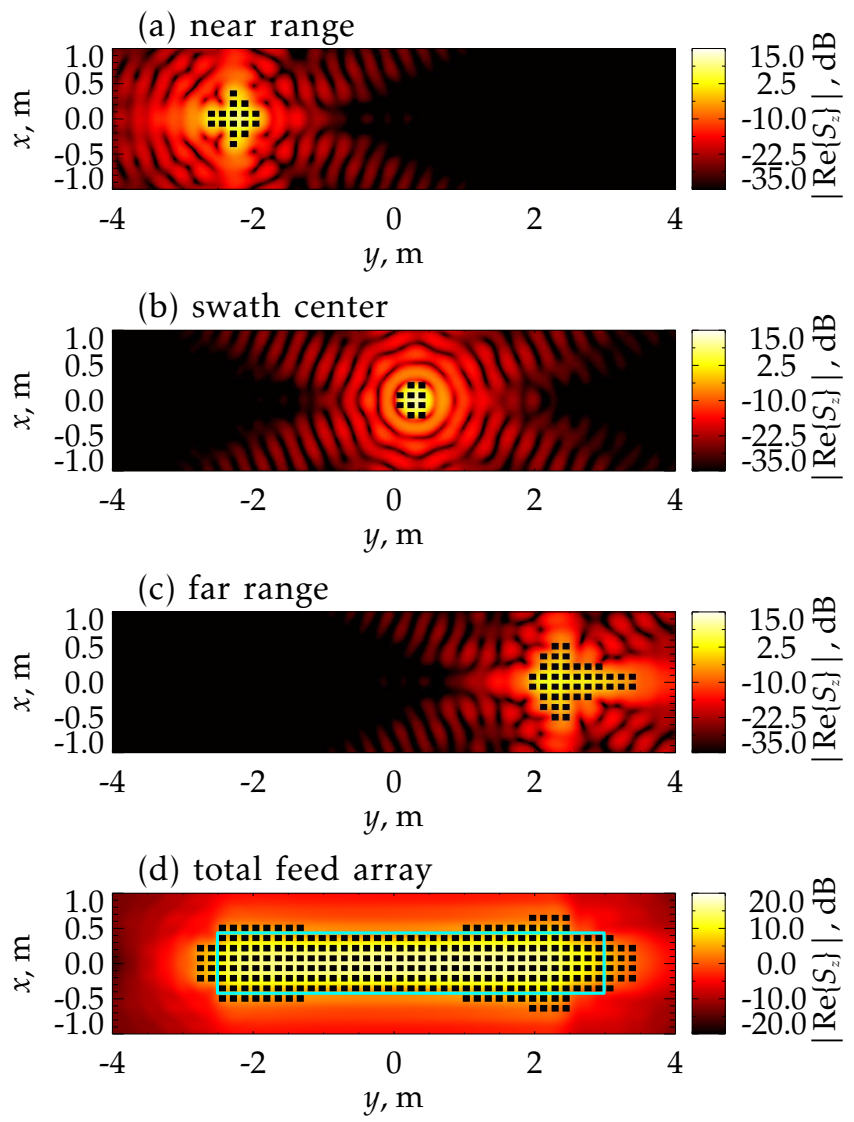

Figure 3: Field in the feed array plane for a plane wave incident from near range (a) swath center (b) and far range (c). The entire feed array (d) results from the combination of the individual feed patches corresponding to the individual plane waves. The blue box indicates the actual feed array size for Tandem-L.

azimuth feed elements have been grouped together. This is explained in more detail in the next subsection. The

\begin{tabular}{l|c} 
frequency & $1.2575 \mathrm{GHz}$ \\
azimuth diameter & $15 \mathrm{~m}$ \\
elevation diameter & $15 \mathrm{~m}$ \\
focal length & $13.5 \mathrm{~m}$ \\
offset (elevation) & $9 \mathrm{~m}$ \\
azimuth elements & 6 \\
azimuth spacing & $0.6 \lambda$ \\
elevation elements & 35 \\
elevation spacing & $0.6591 \lambda$ \\
feed array size & $5.5 \mathrm{~m} \times 0.86 \mathrm{~m}$
\end{tabular}

Table II: Antenna and feed array design parameters using a parabolic reflector type with circular aperture.

design approach according to equation (1) is based on an integration of the focal plane field over a flat surface.

Figure 4 shows the geometric conditions of the Tandem-L SAR antenna in a cross-sectional view. The feed array at the bottom of the image is mounted on top of the satellite bus and connected via a boom with the reflector. The choice for the offset is driven on the one hand by the need of minimizing blockage, which 


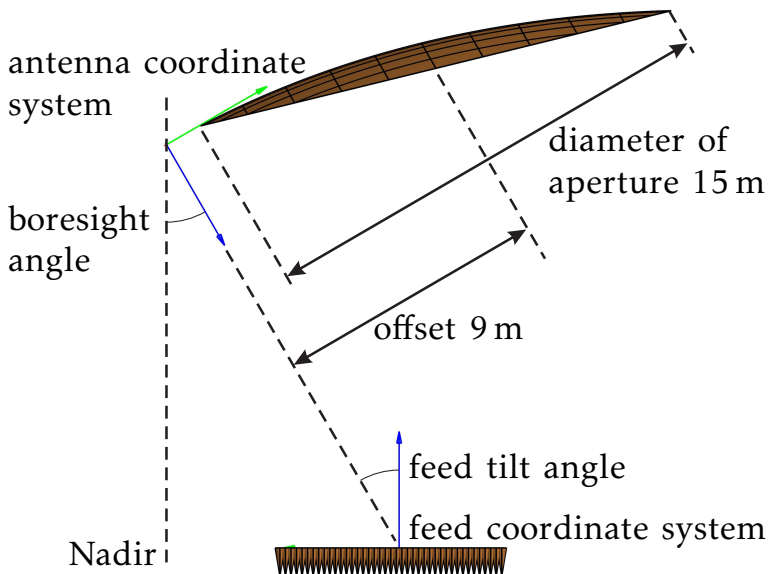

Figure 4: Cross sectional view of the Tandem-L SAR antenna. The flight direction is into the paper plane.

requires large offsets, and on the other hand by avoiding excessive defocusing of the beams, which would be optimal for zero offset. Although a heuristic value, the $9 \mathrm{~m}$ offset turns out to be a good compromise for the given reflector dimensions, feed size and focal length. The focal length is the distance between the origins of the two coordinate systems in Fig. 4. The significance of this compromise becomes evident, by considering a center-fed configuration with zero offset. In this case the reflector aperture would be centered around the boresight axis and the feed array would face the reflector with a feed tilt angle of $0^{\circ}$ causing a maximum of blockage. On the other hand, increasing the offset would shift the reflector further to the right and consequently require an even larger feed tilt angle. This would be best in terms of blockage but result in severe defocusing effects. From a mechanical point of view, the antenna assembly as drawn in Fig. 4, with the feed array horizontally aligned, is usually preferable over tilted ones, since here the system requirements for active attitude maintenance can be significantly reduced ${ }^{3}$. This situation occurs when the boresight angle approximately equals the feed tilt angle. For Tandem- $\mathrm{L}$ these angles will lie in the order of $30-37^{\circ}$.

\section{B. Azimuth Beamforming Concept}

Tandem-L shall acquire SAR data with azimuth resolution down to $7 \mathrm{~m}$. A design goal for the SAR antenna was to cover the required Doppler band with a single azimuth beam, shaped for improved ambiguity performance. As indicated in Fig. 5, the Tandem-L feed array employs three patch doublets in azimuth. Within each doublet the two patch radiators are connected via an RF circuit such that the resulting cross polar patterns in the horizontal and the vertical polarization channel are reduced [21], [22]. Figure 6 shows in the top left an example of a co-polar pattern for horizontal

\footnotetext{
${ }^{3}$ In general the entire mass distribution in the spacecraft needs to be taken into account.
}
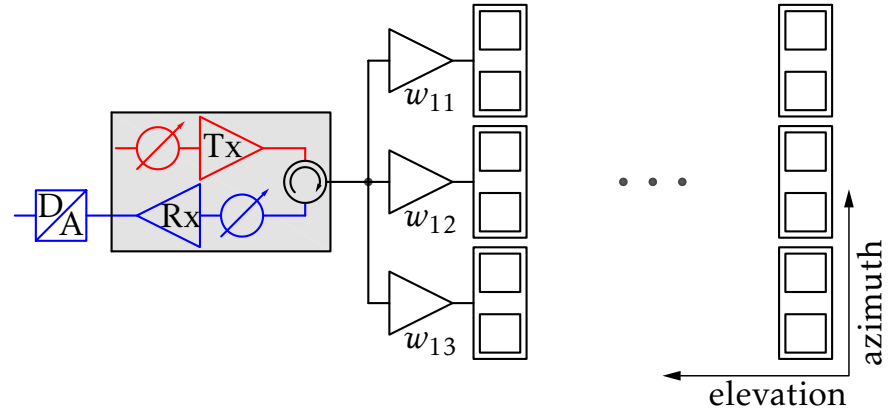

Figure 5: Basic hardware architecture of the feed array. The fixed complex weights $w_{i k}$ shall be realized via different cable lengths and power dividers/combiners.

polarization and in the top right image the corresponding cross-polar pattern. The co-polar-to-cross-polar ratio, measured from peak-to-peak, is $25.6 \mathrm{~dB}$. Important to mention here is that these patterns are secondary patterns, namely the patterns of the primary feed field, scattered by the reflector. The particular beam here corresponds to a patch element in the corner of the feed array and points to near range. Note that elevation and azimuth are the angles in the antenna coordinate system (cf. Fig. 4). Correspondingly, in the second row of Fig. 6, the patterns of a patch-doublet with cross-pol cancellation network is presented. Here, the peak copolar-to-cross-polar ratio improves by $10.2 \mathrm{~dB}$ to $35.9 \mathrm{~dB}$. As explained in more detail in the next pargraph, the three azimuth doublets are combined via fixed weights $w_{i k}, i \in[1,35], k \in[1,3]$ (see Fig. 5). The weights apply on transmission as well as reception, and will be realized by using different cable lengths for phase adjustments, and combiners/dividers for amplitude adjustments. The impact of this pre-weighting on the co- and cross-polar patterns can be studied in the lower row of Fig. 6. The peak co-polar-to-cross-polar ratio improves slightly by another $1 \mathrm{~dB}$ to $36.9 \mathrm{~dB}$, which is not surprising, since the co-polar patterns of the three patch doublets are combined optimally in terms of directivity. But even more important is that an additional notch in azimuth becomes noticeable, which would not be present without this cancellation technique. This notch will be preserved even after applying beamforming in elevation, which means that the notch is scanned over the swath in accordance with the co-polar beam. It turns out that the SAR cross-talk performance predominantly benefits from this feature, since any target for a given range will fall into this notch and therefore contribute with a much reduced cross-talk to the polarimetric polarization channels.

1) Antenna Pattern Optimization: The necessity for optimizing ambiguity performance of the azimuth pattern emerges from the need to vary the pulse repetition interval (PRI) using the Staggered SAR methodology to overcome the problem of the blind ranges, as described in Section IV: Staggered SAR operation with a reasonable quad-pol azimuth ambiguity ratio is only possible with 

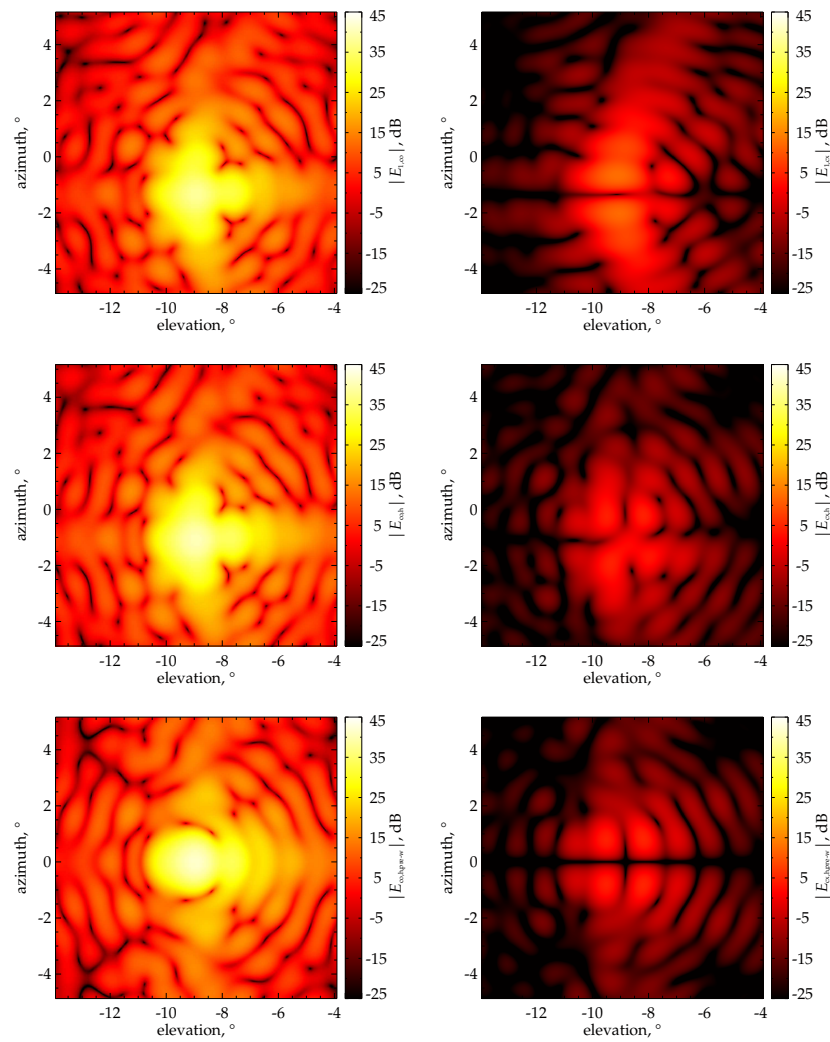

Figure 6: Left column: Secondary Co-polar patterns for h-polarization. Right Column: Corresponding crosspolar patterns. Top row: Patterns for a single patch element (peak co-polar-to-cross-polar ratio $25.6 \mathrm{~dB}$ ). Middle row: Patterns for a patch doublet (peak co-polar-tocross-polar ratio $35.9 \mathrm{~dB}$ ). Lower row: Patterns after preweighting of the three patch doublets (peak co-polar-tocross-polar ratio $36.9 \mathrm{~dB}$ )

narrow azimuth beams that reduce the ambiguous signal energy that would arise from the flanks of the nonoptimized azimuth pattern. This is most critical for the near and far range beams, which naturally tend to broaden due to their distance from the focal point of the feed array. In this context, a three-step procedure for finding optimized azimuth pre-weighting coefficients $w_{i k}$ (cf. Fig. 5) was adopted. The evolution of the weights during this procedure is illustrated in Fig. 7a. Here, the index ' $h$ ' shall indicate that the weights are different for the two polarizations, since the corresponding patterns usually differ slightly in amplitude and phase from each other. Note that in this unwrapped representation the coefficients of channel 36 to 70 correspond to the center column of the feed $(k=2)$, while the other coefficients are associated with the outer azimuth columns $(k=1$ and $k=3$ ). Since the antenna geometry is symmetric, so are the azimuth coefficients. A good result to sharpen the flanks of the azimuth beams could be achieved by placing nulls in the antenna diagram close to the main beam using so called Linear Constrained Minimum Variance beamforming (LCMV) [16], [23]. The corresponding

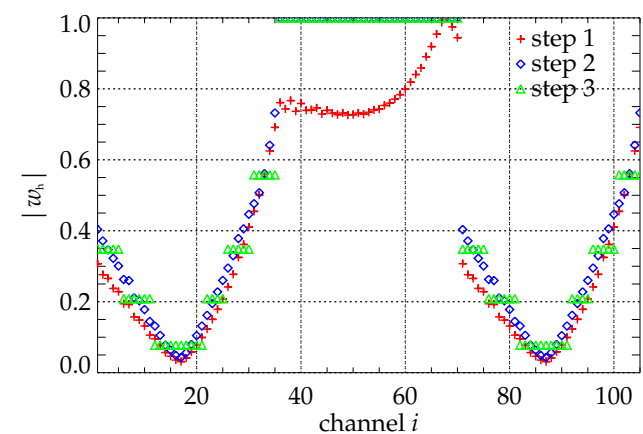

(a)

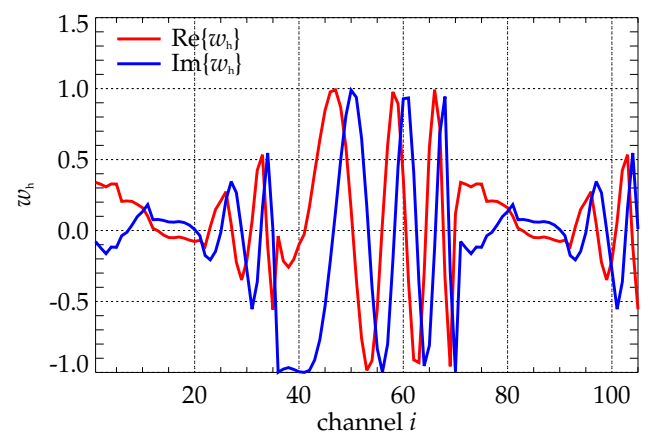

(b)

Figure 7: (a) Absolute value of the pre-weighting coefficients in azimuth. First, LCMV coefficients are computed for every azimuth triplet. Then, these coefficients are normalized to the maximum. Finally, the coefficients are quantized in order to restrict the number of power divider/combiner ratios to four. (b) Real and imaginary part of the final pre-weighting coefficients.

weights are marked by the red '+'-symbols in Fig. 7a. An example of such an azimuth beam is presented in Fig. 8a versus azimuth angle. These patterns correspond to a center feed row $(i=16)$. Clearly, the nulls, which have been placed at $\pm 1.3^{\circ}$ in the co-pol patterns, can be observed. The second step in the optimization procedure is simply a normalization of each of the weight triplets to their maximum, in Fig. 7 indicated by the blue diamonds. In the final step (green triangles) the weights are quantized such that only four discrete combiner/divider ratios are necessary. This step is purely motivated by cost saving arguments. Figure $8 \mathrm{~b}$ shows the beam as presented in Fig. 8a but now with quantized coefficients. Obviously, the deep nulls vanish, however, the narrow beam width is very well preserved. Figure $9 \mathrm{~b}$ shows the azimuth pattern cuts corresponding to the 35 elevation channels after LCMV pre-weighting. These azimuth cuts have been taken at the gain maxima. For comparison, Fig. 9a shows the corresponding azimuth pattern cuts for the center column of the feed array. It becomes clear that this strong variation of the azimuth beamwidths across the swath would prevent achieving the performance requirements in terms of azimuth ambiguities. 


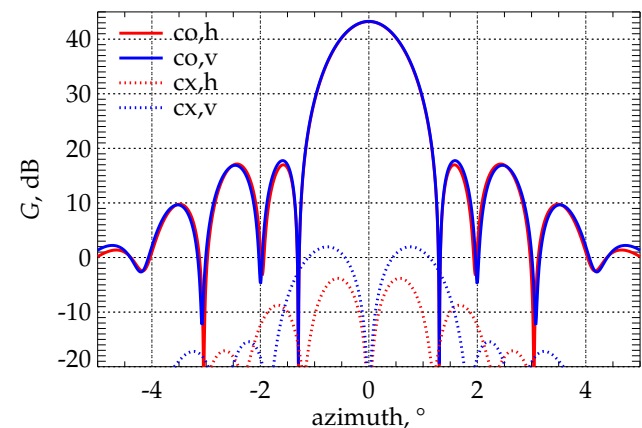

(a)

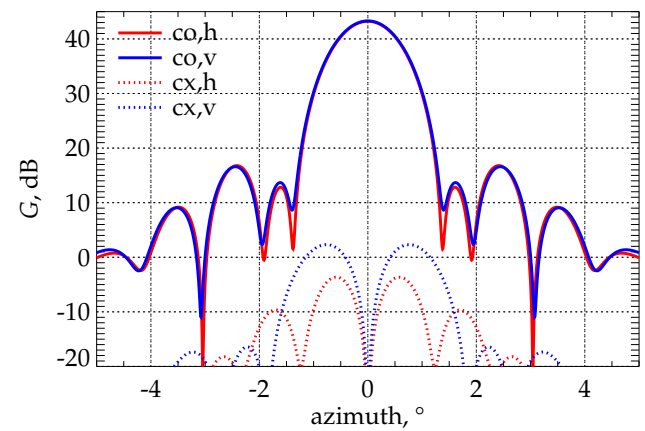

(b)

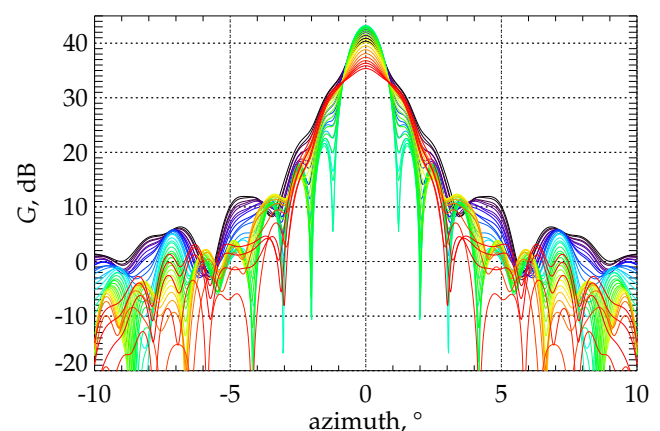

(a)

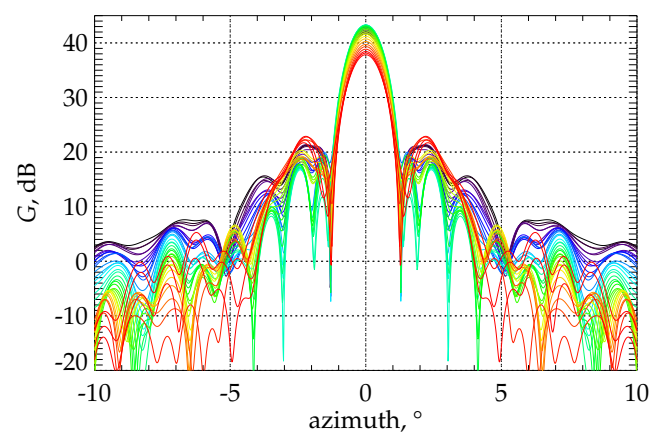

(b)
Figure 8: (a) Azimuth antenna pattern cut after application of LCMV coefficients to the triplet. (b) The same pattern using quantized coefficients.

As mentioned above, on transmit a wide beam is generated by activating all elevation channels at once. However, the azimuth beamforming procedure, which is individual for each elevation segment, has the consequence that the transmit beam cannot simply be generated by activating the 35 elevation channels without proper phase control. Therefore, as last step, the transmit pattern has been optimized using only the phases of the excitation coefficients. This allows achieving a nice table-top shape, which is characteristic of large array-fed reflectors.

\section{Elevation Beamforming Concept}

Sofar the design of the Tandem-L SAR antenna, including the analog feed network, has been discussed. At this point 35 elevation channels are available for each polarization. The most simple solution would be to downlink these 35 channels to ground. However, this is not an option for Tandem-L due to the limited data link capacity. Consequently, processing on board the spacecraft is needed, preferably in the digital domain, since this would allow for flexible re-configuration of the DBF processors. With regard to the beamforming strategy two basic considerations have been taken into account: First, in order to efficiently use the large reflector aperture, the beamforming network will have to be time-variant, what is known as Scan-On-Receive (SCORE) capability
Figure 9: Azimuth cut plots of the antenna patterns taken at the maximum of the 35 reflector beams. (a) Center column of the feed array. (b) Feed array after preweighting.

in the literature. Basically, this means a high receive gain beam tracks the radar echo on ground. Such a concept could be realized by weighting each elevation channel in a time-variant manner with a single complex coefficient and summing up the data streams. However, a second consideration suggests that beamforming via a timevariant filter based DBF structure could improve the SAR imaging performance even more. This is motivated by the fact that the SAR antenna patterns show a distinct non-constant behaviour over the frequency band, which could be accounted for with such a DBF filter structure. A second benefit of this DBF filter structure is that it can deal with long duration pulses, which might relax the pulse peak power.

In Fig. 10 a schematic of the elevation beamforming architecture on receive is shown. The bright yellow beam indicates the broad transmit pattern using all elevation feed elements. On receive $N$ frequency adaptive beams (four to five beams in case of Tandem-L), here illustrated by the rainbow color code, shall be formed. These receive beams simultaneously track the $N$ SAR pulse echoes of length $\tau_{\mathrm{p}}$ traveling over ground. A consequence of the imaging geometry is that the illumination of the echo spectrum is spread in spatial (angular) domain in near range. This problem could be dealt with using more frequency-adapted beams compared to far range. The 

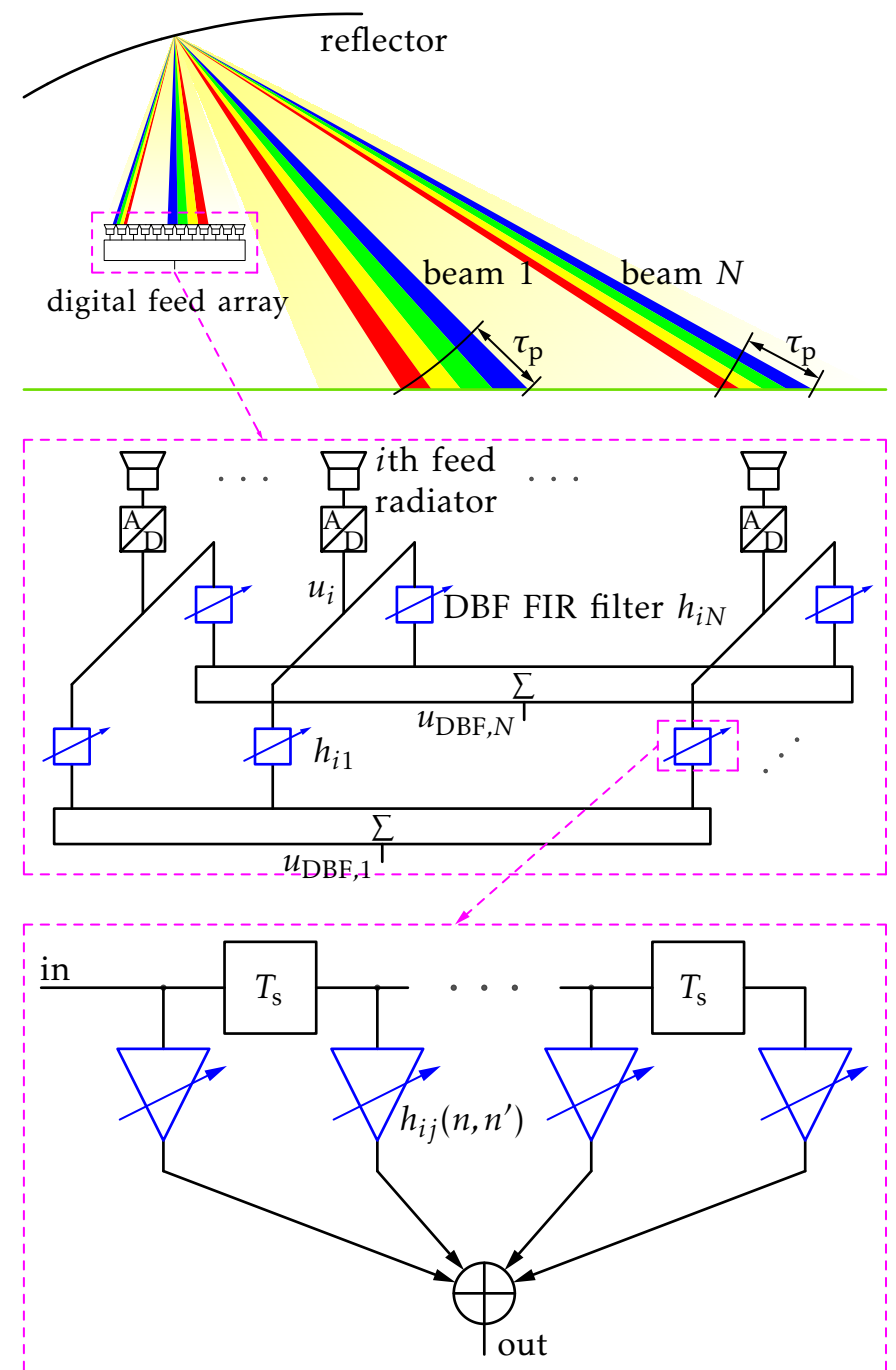

Figure 10: Hardware concept of the receive chain for beamforming in elevation. DBF stands for Digital Beamforming and FIR for Finite Impulse Response.

digital receiver hardware of the feed array is shown in the first zoom window in the middle of Fig. 10. Here, all T/R-modules, amplifiers, I/Q demodulators and filters have been omitted in order to maintain a clear representation. The functionality can be summarized in the following way: The electromagnetic signal received by the $i$ th elevation feed radiator ${ }^{4}$ is digitized by an analogto-digital converter (ADC) and distributed to $N$ finite impulse response (FIR) filters corresponding to the $N$ beams simultaneously scanning the swath. Note, here the count variable for the beams is $j$. The purpose of the FIR filters is to decompose the chirp signal of bandwidth $B$ into several sub-bands and apply a complex weight $w_{i j}$, which is time variant, to each sub-band beam [15], [16], [20]. The structure of such a FIR filter is sketched in the lower right box with the time variant filter coefficients $h_{i j}\left(n, n^{\prime}\right)$. In this context $n$ represents the discrete time

\footnotetext{
${ }^{4}$ For Tandem-L each feed radiator represents a set of pre-weighted patch doublets as sketched in Fig. 5.
}

variable sampled with the frequency $f_{\mathrm{s}}=T_{\mathrm{s}}^{-1}$. Finally, the individual signals have to be summed in order to form the beam. Mathematically, the formation of the $j$ th beam, $j \in[1, N]$, can be described via a convolution of the $i$ th elevation signal $u_{i}$, which is the signal after A/D conversion, with time variant filter coefficients $h_{i j}\left(n, n^{\prime}\right)$.

$$
\begin{aligned}
& u_{\mathrm{DBF}, j}(n)=\sum_{i} \sum_{n^{\prime}=0}^{N_{\mathrm{coef}}-1} u_{i}\left(n-n^{\prime}\right) h_{i j}\left(n, n^{\prime}\right), \\
& h_{i j}\left(n, n^{\prime}\right)=\sum_{m=0}^{M-1} w_{i j}(n, m) \bar{h}\left(n^{\prime}, m\right) .
\end{aligned}
$$

In this context the filter $\bar{h}$ represents a bandpass ${ }^{5}$ which decomposes the chirp signal into $M$ sub-bands. This means that theoretically at every time instance $M \cdot N$ beams are formed. The advantage of such a beamforming concept becomes quite evident by the following consideration: Suppose the SAR pulse has the length $\tau_{\mathrm{p}}$ as drawn in Fig. 10. If one assumes now a very large antenna aperture, such that the beam would have the width of, say, the yellow sub beam, then it is clear that large parts of the pulse energy would be lost. Dividing the pulse into several sub-pulses, as suggested above, basically removes this problem. Moreover, due to the linear relationship of time and frequency for a chirp, it is possible to weight each sub-pulse according to the frequency behaviour of the antenna patterns as well as the receive chain transfer functions in their respective sub-bands.

Not drawn in Fig. 10 is the so called re-formatting and data volume reduction unit. The purpose of this unit is to concatenate and re-formate the $N$ beams to a 2-D data field which can be processed by the data reduction filter as explained in detail in section IV-B.

Tandem-L will rely on conventional T/R-module technology. This means each elevation channel employs a TRM for $\mathrm{v}^{-}$and for h-polarization. A potential solution for future SAR systems could be to split up the transmit and receive chain already after the circulator. In this way the transmit power could be provided by a single source like a traveling wave tube (TWT) or multiple high power amplifiers (HPA) and distributed across the feed array. This power distribution would not necessarily be homogeneous. For example with array-fed reflectors it could make sense to distribute more energy to the feed elements corresponding to far range where the worst NESZ can be expected.

\section{Staggered SAR Operation}

The Tandem-L requirements of Table I are met by operating the instrument in staggered SAR mode [24][26]. The large reflector antenna with the digital feed array shall illuminate on transmit a wide swath and form on receive multiple narrow elevation beams, which

\footnotetext{
${ }^{5}$ The number of FIR filter coefficients $N_{\text {coef }}$ might lie in the order of a few tens.
} 
follow the radar echoes while they traverse the swath. Moreover, the pulse repetition interval $(P R I)$ is continuously varied to overcome the problem of the blind ranges and some additional onboard processing is performed to reduce the amount of data to be downlinked.

\section{A. Concept}

In a multiple elevation beam SAR with constant PRI blind ranges are present between adjacent subswaths, as the radar cannot receive while it is transmitting (Fig. 11a) [17]. If the PRI is continuously varied, even in a cyclical manner, i.e., repeating a sequence of PRIs, there will still be ranges, from which the echoes are not received, because the radar is transmitting. In general, however, those ranges will be different for each transmitted pulse (Fig. 11b). If the PRIs of the sequence are selected such that two consecutive samples in the azimuth direction are never missed and the signal is averagely oversampled in azimuth, it is possible to accurately interpolate the data on a uniform grid and obtain a high resolution SAR image over a wide continuous swath [24]-[26]. As detailed in [26], [27], where demonstrations (a)

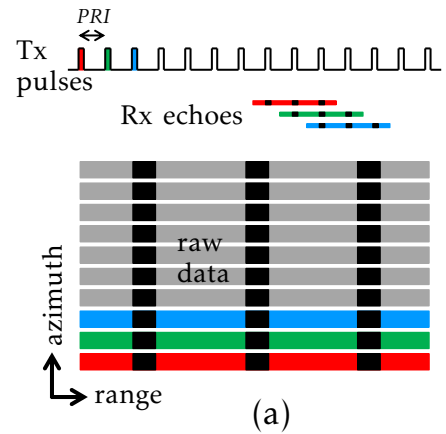

$P R I_{0} P R I_{1} P R I_{2} P R I_{0} P R I_{1} P R I_{2}$

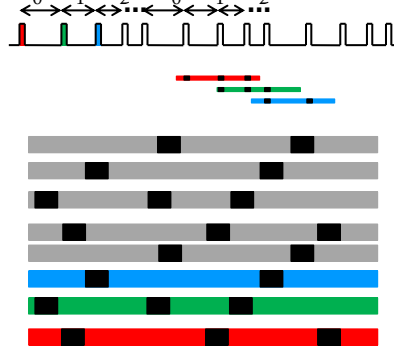

(b)
Figure 11: Location of blind ranges: (a) Constant PRI SAR, (b) Staggered SAR.

with real data are also shown, if the oversampling rate is sufficiently high, as is the case for the Tandem- $\mathrm{L}$, the interpolation is very accurate and independent of the target (i.e., it is very accurate even for a distributed target). The interpolation method only exploits the correlation induced by the azimuth antenna pattern and not the target correlation. This is very important as the proposed system is not intended to image only pointtargets or extended man-made targets, but should also perform well for interferometry and retrieval of other information.

Measures based on real airborne (F-SAR) data show average relative interpolation errors below $-22 \mathrm{~dB}$ in the raw data of a typical scene. These interpolation errors become manifest as azimuth ambiguities in the focused SAR image with an azimuth ambiguity-to-signal ratio in the order of $-28 /-30 \mathrm{~dB}$ [27]. Moreover the azimuth ambiguities are smeared and therefore are likely to be for the same ambiguity level less critical for most parameter retrieval applications. The mainlobe of the impulse response is identical as in a SAR with constant $P R F$ and appropriate sampling.

1) Sequences of PRIs: The selection of the sequence of PRIs to be adopted in a staggered SAR system has been discussed in detail in [26]-[28], where several approaches to sequence design are considered. Specifically, formulas are provided to obtain sequences where two consecutive samples in azimuth are never missed in the raw data. In particular, the use of the more elaborated sequences of PRIs described in [26] is adopted for the staggered SAR modes of Tandem-L. Figure 12 shows the sequence of 224 PRIs to be used for the single- and dual-pol modes of Tandem-L. The mean PRI is $0.385 \mathrm{~ms}$, corresponding to a mean PRF of $2600 \mathrm{~Hz}$.

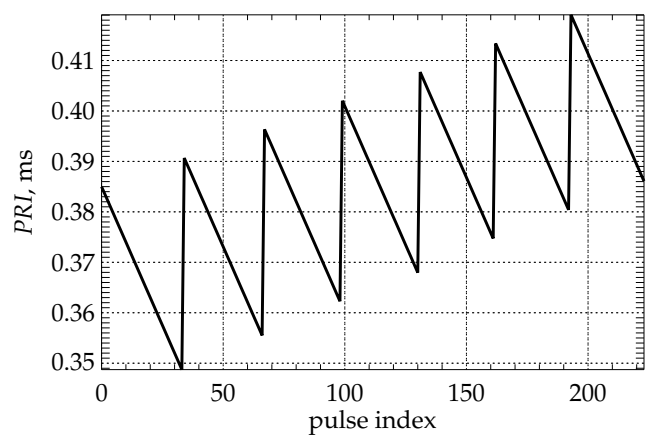

Figure 12: Sequence of PRIs to be used for the singleand dual-pol modes of Tandem-L.

2) Interpolation: Besides the design of the sequence of PRIs, staggered SAR requires additional processing steps that are essential with respect to the ambiguity performance and the amount of data to be downlinked. From a conceptual point of view, the non-uniformly sampled azimuth signal is first resampled to a uniform grid and then focused using a conventional SAR processor. Moreover, it has been shown that resampling data before range-compression leads to better performance than resampling range-compressed data [25].

As far as the resampling method is concerned, Best Linear Unbiased (BLU) interpolation accounts for the statistical properties of the raw azimuth signal and makes use of the knowledge of its Power Spectral Density (PSD) to reconstruct the signal at the desired locations, leading to superior performance compared to two-point linear interpolation, i.e., a weighted average of the closest preceding and succeeding complex samples [24], [26]. As explained in [24], each sample of the uniform grid is obtained as linear combination of a small set of (neighboring) samples of the non-uniformly sampled azimuth signal, where the weights of the linear combination are related to the autocorrelation of the azimuth signal, i.e., the inverse Fourier transform of the antenna power pattern in azimuth.

As discussed in the following in more detail, performing the interpolation on-board allows a considerable 
reduction of the data volume to be transferred to the ground segment.

3) Performance: The main advantage of a staggered SAR over a multiple elevation beam SAR with constant $P R I$ is the possibility to map a wide continuous swath rather than several subswaths mutually separated by blind areas. The attained range and azimuth resolutions are the same as for a multiple elevation beam stripmap SAR with constant PRI.

The NESZ is the same as for a multiple elevation beam SAR with constant $P R I$ - assuming the same imaging parameters and a PRF equal to the mean PRF $(\overline{P R F})$ of the staggered SAR system - but for a possible NESZ degradation. This NESZ degradation is due to the described interpolation, where noise is amplified in the matrix inversion (a specific step in the interpolation algorithm). Taking into account the possible NESZ degradation due to the noise amplification caused by missing samples and azimuth resampling, it holds

$$
N E S Z=N E S Z_{\text {uni }} \cdot \Phi,
$$

where $N E S Z_{\text {uni }}$ is the NESZ of the corresponding constant PRI SAR and $\Phi$ is the SNR scaling factor, i.e., the $S N R$ degradation due to the resampling. An SNR scaling factor larger than 1 indicates that the SNR degrades with respect to the SNR of the corresponding constant PRI SAR. The SNR scaling factor can be assessed by simulation, as explained in the following, and is expected to vary across the swath, as different samples are missing at different ranges. In particular, noise-only raw data can be simulated for both the staggered SAR raw data and the reference raw data with constant PRF equal to the mean PRF on transmit of the staggered SAR. These noiseonly raw data are then processed as the raw data of a point-like scatterer (i.e., resampling to a uniform grid, conventional SAR processing). For both raw (input) and focused (output) data of both the staggered SAR system and the reference system with constant PRF, the SNR is evaluated as the ratio of the energy of the data for a point-like scatterer and the noise-only data. The SNR scaling factor $\Phi$ is then evaluated as the ratio of the ratios of the output and input SNRs of the staggered SAR data and the reference data with constant PRF. Typical values of the SNR scaling factor vary from $0.4 \mathrm{~dB}$ to $0.7 \mathrm{~dB}$ [26], [27].

Staggered SAR operation has significant effects on range and azimuth ambiguities, as discussed in [26], [29]. In a SAR system with constant PRI, during the acquisition of the raw data, the range ambiguous echoes of a scatterer are located at the same ranges along the whole synthetic aperture. This is due to the constant time distance to preceding and succeeding pulses. These echoes cause, after azimuth focusing, the presence of ghost targets in the SAR image, because the ambiguous energy is integrated along azimuth, even though the range migration is not fully matched, as for the scatterer. In a staggered SAR system, the range ambiguities are located at different ranges for different range lines, as the time distance to the preceding and succeeding pulses continuously varies. The ambiguous energy is therefore incoherently integrated and spread almost uniformly across the Doppler spectrum. If the mean PRF of the system is much larger than the processed Doppler bandwidth (PBW) $B_{\mathrm{p}}$, a significant amount of the ambiguous energy is thus filtered out during the SAR processing. Moreover, the residual ambiguous energy of a scatterer is spatially almost uniformly distributed over the whole synthetic aperture and over a range equal to the PRI span times half the speed of light. The same applies to nadir echoes, which result from the same phenomenon [27].

These peculiarities, as well as the specific sequence of PRIs, have to be taken into account for the evaluation of the range ambiguity-to-signal ratio $(R A S R)$. In particular, for a given sequence of PRIs, the RASR has to be evaluated for each of the $M$ transmitted pulses of the sequence. Due to the uniform distribution of the ambiguous energy, the $R A S R$ is then obtained for each slant range by averaging the RASR obtained for the $M$ transmitted pulses. Moreover, the amplitude weighting of the Doppler spectrum applied in the processing $Q(f)$ has to be accounted for. The RASR can be then expressed as [29]

$$
\begin{aligned}
& R A S R \cong \\
& \frac{\sum_{m=0}^{M-1} \sum_{j=1}^{N_{\mathrm{a}}}\left(\frac{\sigma_{0}\left(\eta_{j m}\right)}{R_{j m}^{3} \sin \eta_{j m}} \frac{\int_{-P R F}^{P R F} / 2}{\overline{P R}} G^{2}\left(\theta_{j m}, f\right) \mathrm{d} f \int_{-B_{\mathrm{p}} / 2}^{B_{\mathrm{p}} / 2} Q^{2}(f) \mathrm{d} f\right)}{M \overline{P R F} \cdot \frac{\sigma_{0}\left(\eta_{\text {main }}\right)}{R_{\text {main }}^{3} \sin \eta_{\text {main }}} \int_{-B_{\mathrm{p}} / 2}^{B_{\mathrm{p}} / 2} G^{2}\left(\theta_{\text {main }}, f\right) Q^{2}(f) \mathrm{d} f}
\end{aligned}
$$

where the subscripts $m, m \in[0, \ldots, M-1]$, refer to the transmitted pulse of the sequence and $j, j \in\left[1, \ldots, N_{\mathrm{a}}-1\right]$, to the $N_{\mathrm{a}}$ ambiguous (preceding and succeeding) returns and where the azimuth ambiguities of the range ambiguities have been neglected. The same formula can be also used for the fully polarimetric case, taking into account that the ambiguous echoes from some of the preceding and succeeding pulses correspond to radiated pulses with different polarizations and using the proper value of $\sigma_{0}(\eta)$ for each contribution.

As far as azimuth ambiguities are concerned, for a staggered SAR system it is not always straightforward to evaluate the azimuth ambiguity-to-signal ratio (AASR) using the azimuth antenna pattern as for a constant PRI SAR. The reason is that the resampling operation may change the shape of the azimuth spectrum of the signal. In order to assess the impact of azimuth ambiguities, however, the acquisition process and the signal processing can be simulated, assuming that only a pointlike scatterer is present in the scene. The focused data obtained from the simulation correspond to the 2-D impulse response function (IRF) of the system, from which several performance parameters - and in particular the integrated sidelobe ratio $(I S L R)$ - can be then evaluated. 
A good estimate of the AASR can be then obtained as the difference of the attained ISLR and the ISLR of a constant PRI SAR with PRF equal to the mean PRF on transmit of the staggered SAR system $\overline{P R F}_{\mathrm{Tx}}$, same values for the other system and processing parameters as the staggered SAR, and an azimuth antenna pattern equal to zero outside the interval $\left[-\overline{P R F}_{\mathrm{Tx}} / 2, \overline{P R F}_{\mathrm{Tx}} / 2\right]$ [29].

As far as the quad-pol staggered SAR operation mode is concerned, merging the complex signals available from both cross-polarized (cross-pol) channels of a fully polarimetric SAR leads to a remarkable improvement in the AASR and RASR of cross-pol data, especially if azimuth phase coding is employed, as highlighted in [28], [30]. Assuming that staggered SAR data are resampled and decimated onboard prior to downlink and that the complex signals available from the hv and vh channels are available at the same time, the AASR and $R A S R$ gain from merging (i.e. averaging) the complex signals available from both cross-pol channels can be evaluated by simulation. The AASR gain from merging is due to the non-stationarity of the azimuth impulse response of staggered SAR and is approximately equal to $2-2.5 \mathrm{~dB}$ for typical quad-pol scenarios. As far as the $R A S R$ gain from merging is concerned, this depends on the coherence $\gamma$ and the relative phase $\phi$ of the two co-pol channels. However, if the azimuth phase code proposed in [30] is employed, merging results in a $3 \mathrm{~dB}$ RASR gain, independently of $\gamma$ and $\phi$.

\section{B. Onboard Data Volume Reduction}

In order to meet the azimuth ambiguity requirement of Tandem- $\mathrm{L}$, a high mean PRF is required for all staggered SAR acquisition modes, which results in an increased volume of data to be downlinked. However, as the mean $P R F$ is much larger than the PBW, the amount of data to be downlinked can be drastically reduced by performing onboard a Doppler filtering followed by decimation, as described in [31].

While this technique can also be adopted for SAR systems with constant PRI, in the staggered SAR case Doppler filtering and interpolation can be jointly performed. As sketched in Fig. 13a, the Doppler filtering should be performed on resampled data, which are obtained from the raw staggered SAR (non-uniformly sampled) data through BLU interpolation. Each sample of the resampled data is obtained as a linear combination of some of the samples of the raw staggered SAR data, while each sample of the filtered data is obtained as a linear combination of some of the resampled data. This means that each sample of the filtered data can be obtained directly as a linear combination of some of the staggered SAR data (Fig. 13b). Moreover, there is no need to compute the samples which would anyway be discarded by the decimation operation. The FIR filter can be designed as a Wiener filter, i.e., exploiting the knowledge of the PSD of the useful and disturbance signals.

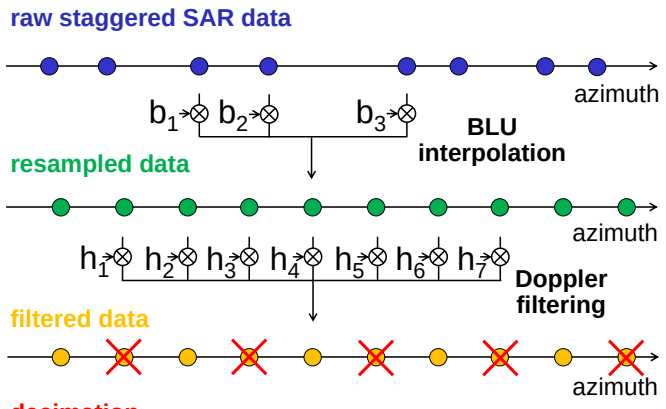

(a)

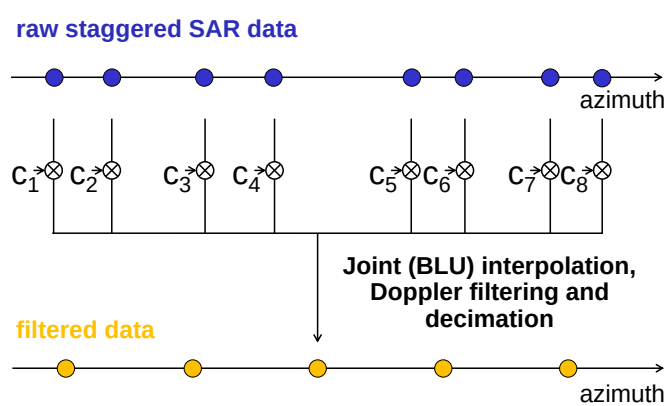

(b)

Figure 13: (a) Interpolation, Doppler filtering, and decimation in the staggered SAR case. (b) Equivalent scheme, where interpolation, Doppler filtering, and decimation are jointly performed. BLU stands for Best Linear Unbiased.

An alternative to design the FIR Wiener filter is given by the Minimum Variance Distortionless Response (MVDR) or Capon beamformer, where only the knowledge of the PSD of the disturbance signal is exploited. The formulas for the design of the filter and the consequent AASR degradation are provided in [31], where it is also shown that a 25-coefficient filter leads to a negligible AASR degradation.

This data volume reduction scheme will be implemented on Tandem- $\mathrm{L}$ in near real-time by means of an onboard hardware. All sets of required (range and azimuth variant) coefficients for joint interpolation, Doppler filtering and decimation are stored on board. For the main single-pol staggered SAR mode it has been estimated that about 10400 distinct sets of 30 coefficients are needed (approximately $0.65 \mathrm{MB}$ ), while the required computational load is well inside the capabilities of state-of-the-art field programmable gate arrays (FPGAs).

\section{SAR Imaging Performance}

The Tandem-L performance has been assessed by means of extensive numerical simulations. This involves the simulation of the SAR raw data signals including the two-way antenna patterns, the application of the digital beamforming techniques in elevation, the staggered SAR reconstruction as well as SAR focusing. Table III collects major parameters describing the imaging geometry and 
the radio frequency (RF) frontend. For an orbit height

\begin{tabular}{l|c}
\hline orbit and Earth parameters & value \\
\hline orbit height at equator & $740465 \mathrm{~m}$ \\
orbit repeat cycle & $8 / 16$ days \\
Earth radius at equator & $6378137 \mathrm{~m}$ \\
surface backscatter model & L-band [32] \\
satellite velocity & $7484 \mathrm{~m} / \mathrm{s}$ \\
\hline RF frontend & value \\
\hline RF center frequency & $1.2575 \mathrm{GHz}$ \\
receiver bandwidth & $85 \mathrm{MHz}$ \\
number of TRMs (h and v-pol) & 70 \\
TRM peak power & $143 \mathrm{~W}$ \\
system noise figure & $3.5 \mathrm{~dB}$ \\
total losses & $3.6 \mathrm{~dB}$
\end{tabular}

Table III: General parameters assumed for the subsequent performance calculations.

of $740 \mathrm{~km}$ and a 16 day repeat cycle the Earth can be mapped using a $175 \mathrm{~km}$ wide swath. This means that with a $350 \mathrm{~km}$ swath, for which the SAR instrument is designed for, every spot on the Earth surface close to the equator can be mapped four times within 16 days.

Before presenting the actual SAR performance, some simulation results shall be discussed. Figure 14 shows an example of the magnitude of a normalized resampled raw data signal $u$ as a function of the azimuth position $y$

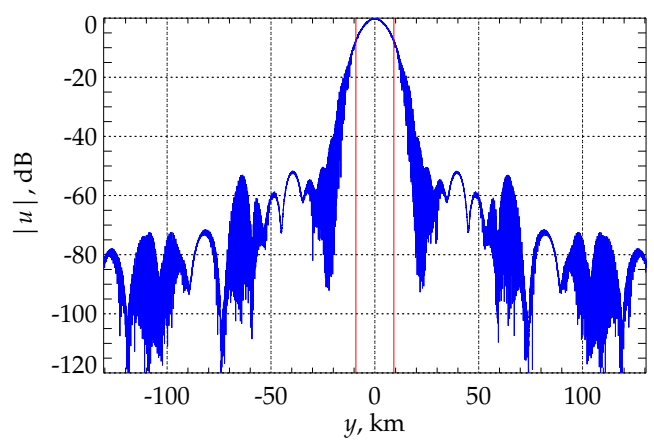

Figure 14: Magnitude of the resampled raw data signal versus azimuth position $y$ for a point target at swath center (ground range $498 \mathrm{~km}$ ). The vertical red lines mark the domain to be processed.

resulting from a point target at the center of the $350 \mathrm{~km}$ swath. The shape is mainly dictated by the two-way azimuth antenna pattern and rather insignificantly by the local incident angle and the range variation. The regions with strong amplitude variation are characteristic for the interpolation process with staggered pulse repetition intervals. Especially the interpolation artifacts at the mainlobe flanks contribute with azimuth ambiguities in the SAR image. The corresponding azimuth focusing filter $h$ is shown in Fig. 15 as a function of the Doppler frequency $f_{\mathrm{D}}$. This filter is constructed according to

$$
h \approx h_{\mathrm{H}} \cdot \frac{g_{\mathrm{Tx}}^{*} \cdot g_{\mathrm{Rx}}^{*}}{a_{\mathrm{Tx}} \cdot a_{\mathrm{Rx}}},
$$

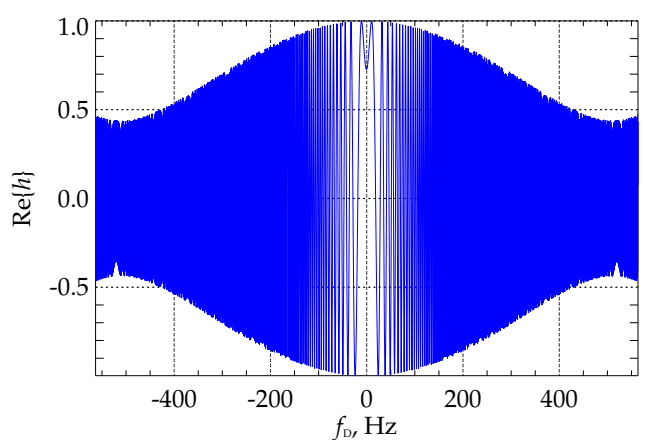

(a)

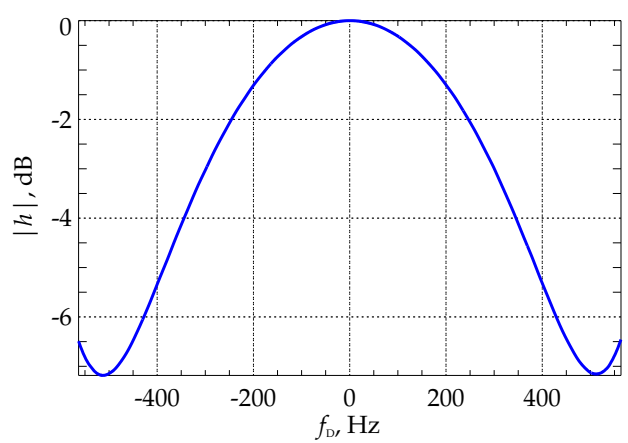

(b)

Figure 15: (a) Real part of the azimuth processing filter versus Doppler frequency for a point target at swath center (ground range $498 \mathrm{~km}$ ). (b) Corresponding power density spectrum.

with the Hamming window $h_{\mathrm{H}}$, the Green's functions $g_{\mathrm{Tx}}$ and $g_{\mathrm{Rx}}$ describing the wave propagation from the transmitter to the target and back to the receiver and the complex antenna patterns $a_{\mathrm{Tx}}$ and $a_{\mathrm{Rx}}$ on transmit and receive. Note that these antenna patterns are understood after digital beamforming in elevation and pre-weighting in azimuth. This filter example is for an azimuth resolution of $7 \mathrm{~m}$ which is equivalent to a processed Doppler bandwidth of $1127 \mathrm{~Hz}$ for the given imaging geometry. Clearly, if the noise spectrum of the SAR raw data was a white circular Gaussian process, after azimuth processing it will be weighted proportional to the filter transfer function. Here, at swath center the high frequency parts of the spectrum would be damped in the order of $7 \mathrm{~dB}$, as it can be seen from Fig. 15b. Finally, the azimuth impulse response function is shown in Fig. 16a and in a zoom view in Fig. 16b. In the zoom view the attenuation of the sidelobes resulting from the Hamming weighting can be observed.

The mode dependent parameters, derived from the requirements in Table I are listed in Table IV. Since staggered SAR needs an over-sampling in azimuth in order to properly reconstruct the signal, the mean PRF has been chosen $2600 \mathrm{~Hz}$ in single- and dual-polarization mode and $2 \times 2100 \mathrm{~Hz}$ in quad-polarization mode. This results in the best compromise between range and azimuth 


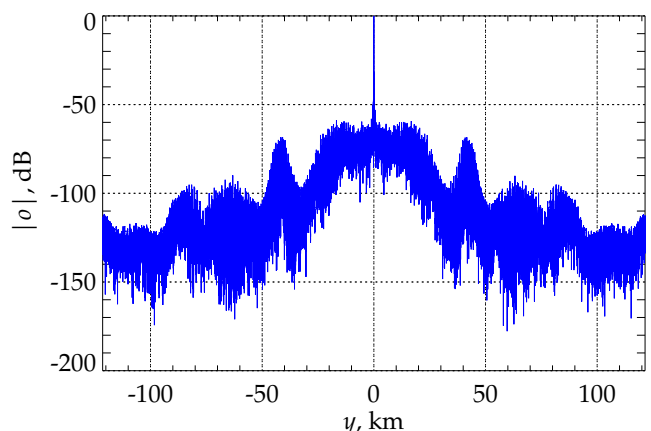

(a)

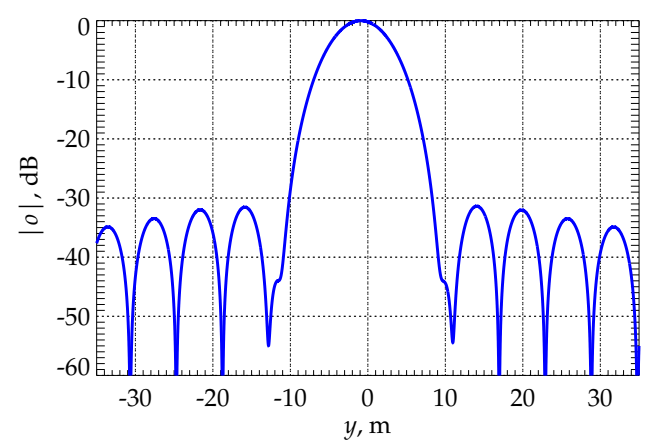

(b)

Figure 16: (a) Focused azimuth SAR signal. The mainlobe of the azimuth impulse response function, lying at $y=$ $0 \mathrm{~km}$, is hardly visible due to the large extent of the $y$ axis. (b) Zoom into the mainlobe region.

\begin{tabular}{l|c|c} 
polarization & single/dual & quad \\
\hline min./max. inc. angle & $26.300^{\circ} / 46.975^{\circ}$ & $28.400^{\circ} / 39.455^{\circ}$ \\
min./max. look angle & $23.390^{\circ} / 40.921^{\circ}$ & $25.224^{\circ} / 34.706^{\circ}$ \\
swath width & $350 \mathrm{~km}$ & $175 \mathrm{~km}$ \\
operation mode & staggered & staggered \\
polarization basis & linear & linear \\
pulse bandwidth & $84 \mathrm{MHz}$ & $84 \mathrm{MHz}$ \\
$\overline{P R F}$ & $2600 \mathrm{~Hz}$ & $2 \times 2100 \mathrm{~Hz}$ \\
pulse duty cycle & $4 \%$ & $8 \%$ \\
azimuth resolution & $7 \mathrm{~m}$ & $7 \mathrm{~m}$ \\
proc. Doppler BW & $1127 \mathrm{~Hz}$ & $1127 \mathrm{~Hz}$ \\
elevation beamforming & MVDR & MVDR \\
azimuth beamforming & pre-weighting & pre-weighting \\
range weighting func. & Hamming $(0.6)$ & Hamming $(0.6)$ \\
azimuth weighting func. & Hamming $(0.6)$ & Hamming $(0.6)$
\end{tabular}

Table IV: Parameters for the main SAR imaging modes.

ambiguities. The resulting performances are shown in Figs. 17 and 18.

\section{Options for Higher Resolution Imaging}

\section{A. Single-/Dual-Pol Modes with $5 \mathrm{~m}$ Resolution}

So far the imaging performance for $7 \mathrm{~m}$ azimuth resolution has been proved. However, there might be a reason in favour for an even better azimuth resolution then $7 \mathrm{~m}$. As shown in Fig. 15b the azimuth processing filter is strongly tapered. The reason for this is that the

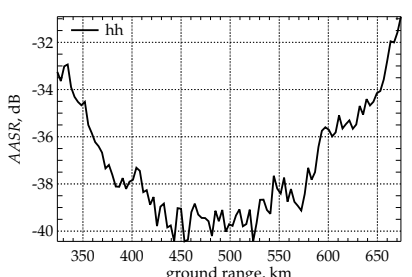

(a)

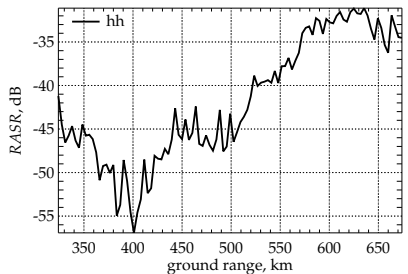

(c)

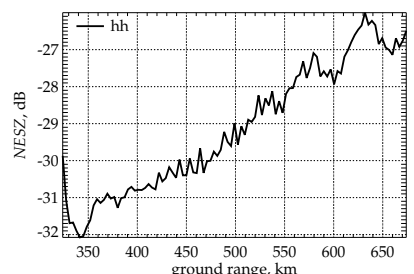

(b)

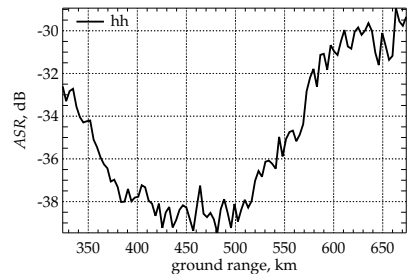

(d)
Figure 17: (a) Azimuth ambiguity-to-signal ratio (AASR) versus ground range for the single-/dual-pol staggered SAR mode as defined in Table IV. (b) Noise equivalent sigma zero (NESZ). (c) Range ambiguity-to-signal ratio $(R A S R)$. (d) Total ambiguity ratio (ASR). Results shown for the hh polarization.

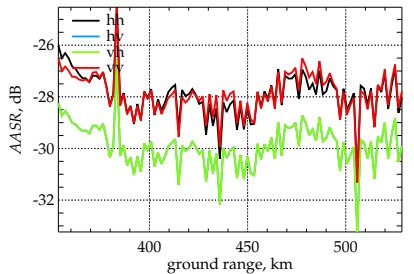

(a)

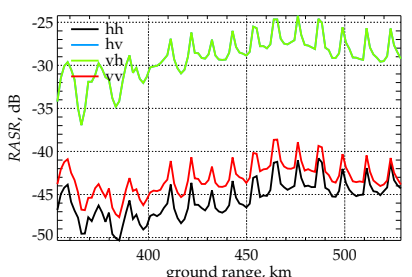

(c)

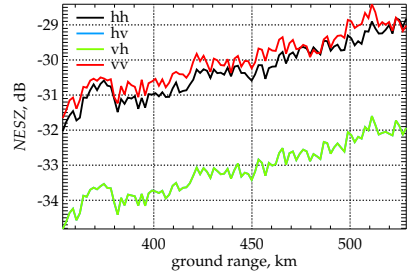

(b)

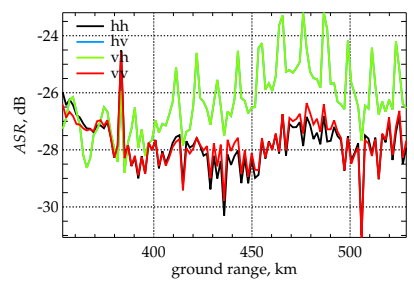

(d)
Figure 18: (a) Azimuth ambiguity-to-signal ratio (AASR) versus ground range for the quad-pol staggered SAR mode as defined in Table IV. (b) Noise equivalent sigma zero (NESZ). (c) Range ambiguity-to-signal ratio (RASR). (d) Total ambiguity ratio $(A S R)$. The cross polarization channels have been merged, as explained above, which helps to improve the cross polar range and azimuth ambiguities.

Hamming window outweighs the two-way pattern by roughly $7 \mathrm{~dB}$. If one is interested in a more uniform noise spectrum it could make sense to process a wider 
Doppler domain. By this the Hamming windowing and the two-way pattern compensation would more or less cancel out. Figure 19 shows a simulation example of a point target at swath center, similar to the one presented in Figs. 14, 15 and 16, except that here the raw data have been processed with $5 \mathrm{~m}$ azimuth resolution, which is equivalent to $1577 \mathrm{~Hz}$ Doppler bandwidth. By

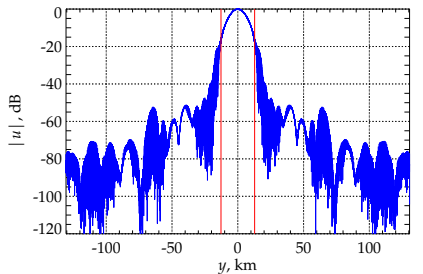

(a)

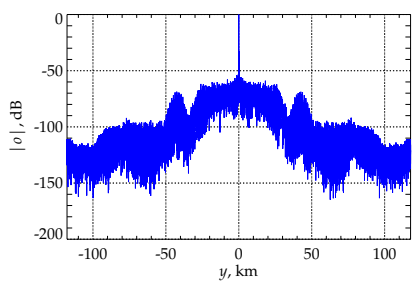

(c)

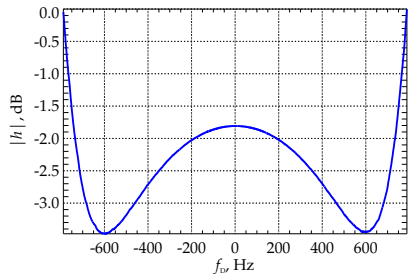

(b)

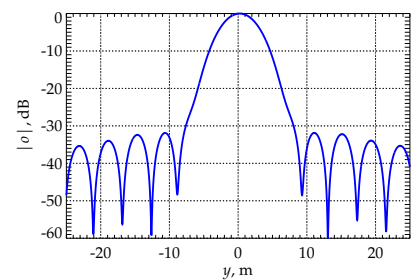

(d)
Figure 19: (a) Simulated raw data signal versus azimuth $y$ for a point target at ground range position at $498 \mathrm{~km}$. The red vertical lines indicate the domain to be processed. (b) Azimuth filter with Hamming weighting and pattern compensation. (c) Azimuth impulse response function. The mainlobe, lying at $y=0 \mathrm{~km}$, is hardly visible due to the large extent of the $y$-axis. (d) Zoom view of the mainlobe region of the azimuth impulse response function.

comparing the azimuth filter spectra from Figs. 15 and $19 \mathrm{~b}$ it becomes clear that in the latter case a much more homogeneous noise spectrum can be expected. The imaging performance as presented in Fig. 20 exhibits a slight degradation in terms of the $A A S R$ by roughly $1.5 \mathrm{~dB}$. The $N E S Z$ degrades by roughly $2.5 \mathrm{~dB}$, therefore the pulse duty cycle has been increased from $4 \%$ to $6 \%$ ( $8 \%$ would be optimal).

\section{B. Spotlight Mode}

Very high azimuth resolutions can be achieved with additional hardware effort, for instance by increasing the feed array in azimuth direction by additional columns [20], [33]. An interesting alternative to this is represented by a concept where the SAR satellite is rotated around its nadir axis. The antenna footprint on ground performs, depending on the rotation rate, a movement similar to that of a windshield wiper. This allows to illuminate a region on ground for a longer period of time as in a conventional spotlight mode. Preliminary investigations showed that the spacecraft needs to be rotated by $720^{\circ}$

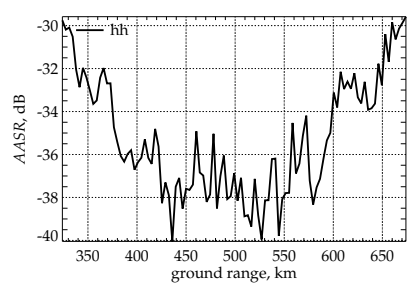

(a)

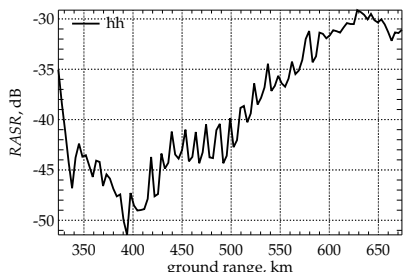

(c)

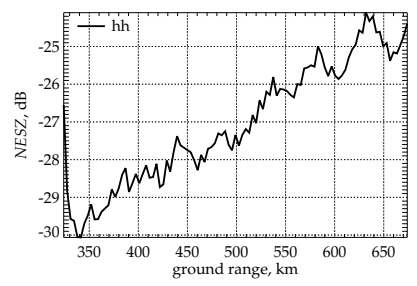

(b)

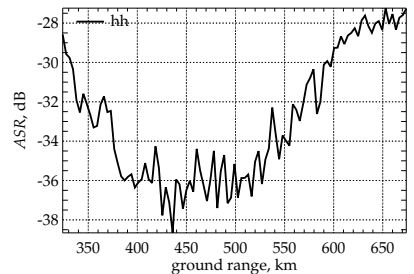

(d)
Figure 20: (a) Azimuth ambiguity-to-signal ratio (AASR) versus ground range for the staggered SAR mode processed with $5 \mathrm{~m}$ resolution. (b) Noise equivalent sigma zero (NESZ). (c) Range ambiguity-to-signal ratio (RASR). (d) Total ambiguity ratio $(A S R)$.

in order to ensure stable SAR acquisition after reaction wheel activity. One challenge for such a mode lies in a proper adjustment of the rotation rate, the PRF and the achievable swath length. Due to this windshield wiper like movement of the antenna footprint it can be expected that targets at varying slant ranges will be illuminated quite differently and therefore the performance along and across the swath might also vary strongly. Additionally, it might be necessary to adapt the scan rate in elevation depending on the azimuth position. Such a $1 \mathrm{~m}$-mode will be investigated in more detail in the next mission phases.

\section{Improved Elevation Beamforming}

This section shifts focus to an elevation beamforming technique which may prove useful to extend the width of the swaths over which the performance requirements are achieved. Even though the technique may be applied regardless of a specific mode, the quad-pol staggered mode of section V described in Table IV is considered as an example of the possible gain in swath width. TandemL's digital receiver hardware, as presented in Fig. 10, is in principle suited for any kind of beamforming technique, since the coefficients for the DBF FIR filters are precomputed on ground and stored in the FPGAs on board the spacecraft.

Fulfilling the performance requirements over a wide swath is especially challenging for quad-pol modes, for which the cross-pol range ambiguity levels become driving factors of the design. This can be visualized using the elevation patterns in Fig. 21a, where the receive SCORE beam formed with MVDR at a look angle of 
$40^{\circ}$ is shown. The signal position is highlighted by a green cross, whereas the position of cross-pol and copol range ambiguities are highlighted by yellow and red crosses, respectively. Note that several positions exist for each order of ambiguity due to the PRI variation. Closer analysis of this pattern illustrates the reason why $R A S R$ performance for the quad-pol modes is often poor in far range: The range ambiguities arise either from the mainlobe or relatively high sidelobes of the pattern. In spite of its robustness and effectiveness in maximizing gain while setting the direction of maximum response, MVDR beamforming offers no direct control over the mainlobe extension or sidelobe levels. A technique presented in [34] as an extension of this beamformer (also known in the literature as sidelobe canceller) allows however introducing a further constraint, namely the maximum level with respect to the main beam over a grid of angles which define the sidelobe region. The resulting problem can be solved numerically with knowledge of the patterns with convex optimization packages. The SCORE pattern obtained by this method is seen in Fig. $21 \mathrm{~b}$, where the sidelobe region - designed to span the position of the range ambiguities in the swath - and the corresponding constraint of a (power) level no higher than $-33 \mathrm{~dB}$ with respect to the main beam are shown by the dashed black line.

As expected, such a design of the sidelobe region allows additional suppression of range ambiguities. In Fig. 21c the RASR levels obtained for the quad-pol mode of section $\mathrm{V}$ with the alternative elevation beamforming are illustrated. The parameters from the third column of Table IV apply, except for the swath limits, which were extended to look angles from $32.2^{\circ}$ to $40^{\circ}$. RASR levels better than $-25.3 \mathrm{~dB}$ could thus be achieved over a swath of circa $325 \mathrm{~km}$, a considerable gain over the $175 \mathrm{~km}$ swath seen before. Figure 21d compares the worst-case RASR levels (HV polarization) with MVDR and the sidelobe-constrained beamformer, highlighting the potential range ambiguity suppression gain of the approach. In this case the full $350 \mathrm{~km}$ swath of the single-pol mode of Table IV is considered.

The results indicate that a $350 \mathrm{~km}$ swath may be expected to be achieved by further optimization of the parameters, using e.g. a slightly lower mean $P R F$ or a more advanced criterion for the sidelobe region design.

Possible disadvantages of this approach include a loss of gain in comparison to a MVDR beamformer using the same number of elevation elements, which, however, can be kept lower than $1 \mathrm{~dB}$ by adequate choice of the sidelobe region. The use of additional elements is also beneficial to the pattern implementation in this case, presenting the opportunity to compensate the gain loss to some extent, at the cost of additional complexity. However, employing patterns with high dynamic range also means that such a beamformer might be sensitive to imprecise pattern knowledge as well as other uncalibrated errors. Therefore, the sidelobe canceller is regarded experimental for Tandem-L. Nevertheless, if

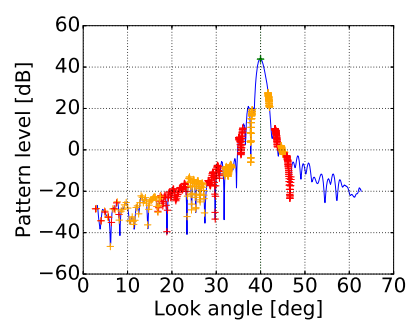

(a)

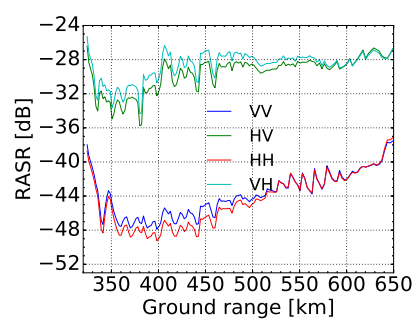

(c)

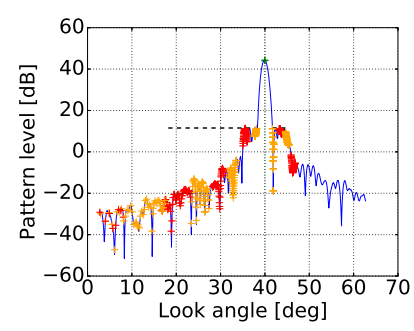

(b)

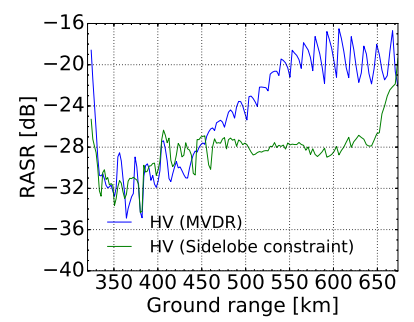

(d)
Figure 21: SCORE beams on far range (look angle of $40^{\circ}$ ) and associated RASR levels. (a) far range SCORE beam obtained by MVDR beamforming with five active elevation elements. (b) far range SCORE beam obtained by the sidelobe-constrained beamformer with eleven active elevation elements. (c) RASR levels obtained for the different polarizations using the sidelobe constraint method in a swath between look angles of $32.2^{\circ}$ and $40^{\circ}$. (d) worst-case RASR (HV polarization) for the MVDR and the sidelobe constrained beamformer over the $350 \mathrm{~km}$ of the single-pol mode of Table IV.

this technique is proven to be stable it might become the baseline beamforming concept.

\section{CONCLUSION}

Gapless SAR acquisitions are an important feature for the scientific user community. Therefore, the TandemL SAR instrument comprises staggered SAR operation as baseline for all imaging modes. This paper addressed major design challenges encountered with this operation principle. Specifically, the need of oversampling the SAR data in azimuth led to a feed array concept, where narrow azimuth beams are generated by pre-weighting pairs of azimuth feed elements. These azimuth feed pairs have been optimized for cross-polar pattern suppression. The Tandem-L SAR instrument is realized as a fully digital, highly integrated system with a digital beamforming unit at its core. This unit allows to cope with a second challenge induced by staggered SAR operation, namely the parallel generation of up to five digital beams, simultaneously scanning the swath. A further feature of this DBF network is the fact that the non-constant frequency behaviour of the SAR antenna can be accounted for using 
frequency dependent beamforming coefficients. Finally, a third issue related to PRI staggering is the large data volume generated as consequence of the required oversampling in azimuth. For this, Tandem-L will employ a specific unit which firstly performs interpolation of the non-uniformly sampled data and secondly decimation.

In the above performance assessment the very basic MVDR beamforming technique has been found optimal. This concept promises the best performance in terms of sensitivity. Although complex pattern information and knowledge of the signal direction is required, this beamforming concept can be regarded as robust. In this context also the potential of higher azimuth resolution modes like five-meter stripmap and one-meter spotlight have been discussed. As shown in the last part of the paper, more advanced beamforming techniques with sidelobe constraints could help to improve the ambiguity performance further.

\section{REFERENCES}

[1] G. Krieger, I. Hajnsek, K. Papathanassiou, M. Eineder, M. Younis, F. D. Zan, P. Prats, S. Huber, M. Werner, H. Fiedler, A. Freeman, P. Rosen, S. Hensley, W. Johnson, L. Veilleux, B. Grafmüller, R. Werninghaus, R. Bamler, and A. Moreira, "The Tandem-L Mission Proposal: Monitoring Earth's Dynamics with High Resolution SAR Interferometry," in IEEE Radar Conference, May 2009.

[2] G. Krieger, I. Hajnsek, K. Papathanassiou, M. Eineder, M. Younis, F. DeZan, P. Lopez-Dekker, S. Huber, M. Werner, P. Prats, H. Fiedler, R. Werninghaus, A. Freeman, P. Rosen, S. Hensley, B. Grafmüller, R. Bamler, and A. Moreira, "Tandem-L: A Mission for Monitoring Earth System Dynamics with High Resolution SAR Interferometry," in 8th European Conference on Synthetic Aperture Radar (EUSAR), Jun 2010, pp. 506-509.

[3] G. Krieger, I. Hajnsek, K. Papathanassiou, M. Eineder, M. Younis, F. D. Zan, S. Huber, P. Lopez-Dekker, P. Prats, M. Werner, Y. Shen, A. Freeman, P. Rosen, S. Hensley, B. Johnson, L. Villeux, B. Grafmüller, R. Werninghaus, R. Bamler, and A. Moreira, "Tandem-L: An Innovative Interferometric and Polarimetric SAR Mission to Monitor Earth System Dynamics with High Resolution," in IEEE International Geoscience and Remote Sensing Symposium (IGARSS), Jul 2010, pp. 253-256.

[4] A. Moreira, G. Krieger, M. Younis, I. Hajnsek, K. Papathanassiou, M. Eineder, and F. D. Zan, "Tandem-L: A Mission Proposal for Monitoring Dynamic Earth Processes," in IEEE International Geoscience and Remote Sensing Symposium (IGARSS), Jul 2011, pp. $1385-1388$.

[5] A. Moreira, G. Krieger, I. Hajnsek, K. Papathanassiou, M. Younis, P. Lopez-Dekker, S. Huber, M. Villano, M. Pardini, M. Eineder, F. D. Zan, and A. Parizzi, "Tandem-L: A Highly Innovative Bistatic SAR Mission for Global Observation of Dynamic Processes on the Earth's Surface," IEEE Geoscience and Remote Sensing Magazine, vol. 3, no. 2, pp. 8-23, Jun 2015.

[6] M. Bachmann, D. B. Tridon, F. D. Zan, G. Krieger, and M. Zink, "Tandem-L Observation Concept - An Acquisition Scenario for the Global Scientific Mapping Machine," in 11th European Conference on Synthetic Aperture Radar (EUSAR), Jun 2016, pp. 1-5.

[7] S. Huber, M. Villano, M. Younis, G. Krieger, A. Moreira, B. Grafmueller, and R. Wolters, "Tandem-L: Design Concepts for a NextGeneration Spaceborne SAR System," in 11th European Conference on Synthetic Aperture Radar (EUSAR), Jun 2016.

[8] M. J. Sanjuan-Ferrer, M. Pardini, D. B. Tridon, P. Lopez-Dekker, K. Papathanassiou, and M. Bachmann, "Product-Level Performance Models for the Tandem-L Mission: Forest Structure Case Study," in 11th European Conference on Synthetic Aperture Radar (EUSAR), Jun 2016, pp. 1-6.

[9] P. Xaypraseuth, R. Satish, and A. Chatterjee, "NISAR Spacecraft Concept Overview: Design challenges for a proposed flagship dual-frequency SAR Mission," in IEEE Aerospace Conference, Mar 2014, pp. 1-11.
[10] I. Hajnsek, M. Shimada, M. Eineder, K. Papathanassiou, T. Motohka, M. Watanabe, M. Ohki, F. D. Zan, P. Lopez-Dekker, G. Krieger, and A. Moreira, "Tandem-L: Science Requirements and Mission Concept," in 10th European Conference on Synthetic Aperture Radar (EUSAR), Jun 2014, pp. 1-4.

[11] “Tandem-L," http://www.dlr.de/hr/en/Portaldata/32/Resources /dokumente/tdml/Tandem-L-Brochure-2016-04.pdf, DLR, Apr 2016.

[12] S. Huber, M. Younis, A. Patyuchenko, and G. Krieger, "A Novel Digital Beam-Forming Concept for Spaceborne Reflector SAR Systems," in European Radar Conference (EuRAD), Oct 2009, pp. $238-241$.

[13] _ _ "Digital Beam Forming Concepts with Application to Spaceborne Reflector SAR Systems," in International Radar Symposium (IRS), Jun 2010, pp. 1-4.

[14] — "Digital Beam Forming Techniques for Spaceborne Reflector SAR Systems," in 8th European Conference on Synthetic Aperture Radar (EUSAR), Jun 2010, pp. 962-965.

[15] S. Huber, "Spaceborne SAR Systems with Digital Beamforming and Reflector Antenna," Ph.D. dissertation, Fakultät für Elektrotechnik und Informationstechnik des Karlsruher Instituts für Technologie (KIT), Feb 2013.

[16] S. Huber, M. Younis, G. Krieger, and A. Moreira, "Advanced Spaceborne SAR Systems with Array-Fed Reflector Antennas," in IEEE Radar Conference (RadarCon), May 2015, pp. 0253-0258.

[17] G. Krieger, N. Gebert, M. Younis, F. Bordoni, A. Patyuchenko, and A. Moreira, "Advanced Concepts for Ultra-Wide-Swath SAR Imaging," in European Conference on Synthetic Aperture Radar (EUSAR), vol. 2, Jun 2008, pp. 31-34.

[18] M. Younis, S. Huber, A. Patyuchenko, F. Bordoni, and G. Krieger, "Performance Comparison of Reflector- and Planar-Antenna based Digital Beam-Forming SAR," International Journal of Antennas and Propagation, vol. 2009, pp. 1-14, Jun 2009.

[19] A. Freeman, G. Krieger, P. Rosen, M. Younis, W. Johnson, S. Huber, R. Jordan, and A. Moreira, "SweepSAR: Beam-forming on Receive using a Reflector-Phased Array Feed Combination for Spaceborne SAR," in IEEE Radar Conference (RadarCon), May 2009.

[20] S. Huber, M. Younis, A. Patyuchenko, G. Krieger, and A. Moreira, "Spaceborne Reflector SAR Systems with Digital Beamforming," IEEE Transactions on Aerospace and Electronic Systems, vol. 48, no. 4, pp. 3473-3493, Oct 2012.

[21] M. Naranjo, A. Montesano, F. Monjas, M. Gomez, R. Manrique, F. Heliere, and K. V. Klooster, "Biomass P-Band SAR Reflector Antenna - Feed S/S Breadboarding," in 10th European Conference on Synthetic Aperture Radar (EUSAR), Jun 2014, pp. 1-4.

[22] P. Valle, G. Orlando, R. Mizzoni, F. Hélière, and K. van't Klooster, "P-band Feedarray for BIOMASS," in 6th European Conference on Antennas and Propagation (EUCAP), Mar 2012, pp. 3426-3430.

[23] H. L. V. Trees, Optimum Array Processing. John Wiley \& Sons, Inc., 2002.

[24] M. Villano, G. Krieger, and A. Moreira, "Staggered SAR: HighResolution Wide-Swath Imaging by Continuous PRI Variation," IEEE Transactions on Geoscience and Remote Sensing, vol. 52, no. 7, pp. 4462-4479, Jul 2014.

[25] —, "A Novel Processing Strategy for Staggered SAR," IEEE Geoscience and Remote Sensing Letters, vol. 11, no. 11, pp. 18911895, Nov 2014.

[26] M. Villano, "Staggered Synthetic Aperture Radar," Ph.D. dissertation, Fakultät für Elektrotechnik und Informationstechnik des Karlsruher Instituts für Technologie (KIT), Feb 2016.

[27] M. Villano, G. Krieger, M. Jäger, and A. Moreira, "Staggered SAR: Performance Analysis and Experiments with Real Data," IEEE Transactions on Geoscience and Remote Sensing, vol. 55, no. 11, pp. 6617-6638, Nov 2017.

[28] M. Villano, G. Krieger, and A. Moreira, "New Insights Into Ambiguities in Quad-Pol SAR," IEEE Transactions on Geoscience and Remote Sensing, vol. 55, no. 6, pp. 3287-3308, Jun 2017.

[29] _ "Ambiguities and Image Quality in Staggered SAR," in IEEE 5th Asia-Pacific Conference on Synthetic Aperture Radar (APSAR), Sep 2015, pp. 204-209.

[30] - "Reconsideration of Ambiguities in Quad-Pol SAR," in 11th European Conference on Synthetic Aperture Radar (EUSAR), Jun 2016.

[31] — , "Onboard Processing for Data Volume Reduction in HighResolution Wide-Swath SAR," IEEE Geoscience and Remote Sensing Letters, vol. 13, no. 8, pp. 1173-1177, Aug 2016. 
[32] F. T. Ulaby and M. Dobson, Handbook of Radar Scattering Statistics for Terrain. Artech House, Inc., 1989.

[33] F. Q. de Almeida and G. Krieger, "Multichannel Staggered SAR Azimuth Sample Regularization," in 11th European Conference on Synthetic Aperture Radar (EUSAR), Jun 2016, pp. 1-6.

[34] J. Liu, A. B. Gershman, Z.-Q. Luo, and K. M. Wong, "Adaptive Beamforming With Sidelobe Control: A Second-Order Cone Programming Approach," IEEE Signal Processing Letters, vol. 10, no. 11, pp. 331-334, Nov 2003.

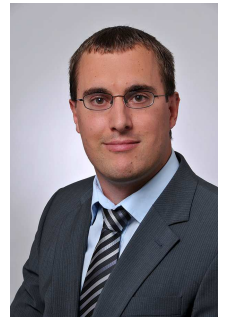

Sigurd Huber received the Dipl.-Ing. (M.S.) and Dr.-Ing. (Ph.D.) (Hons.) degrees in electrical and communication engineering from the Technical University of Munich (TUM), Germany, in 2005 and the Karlsruhe Institute of Technology (KIT), Germany, in 2014, respectively.

Since 2005 he is with the Microwaves and Radar Institute of the German Aerospace Center (DLR), Oberpfaffenhofen, Germany, where he is engaged in the conception of future synthetic aperture radar systems, utilizing advanced multichannel antenna architectures. He is involved in national and international projects in the field of Earth observation, encompassing cooperations with industry partners (Airbus, OHB), as well as research organizations (ESA, NASA/JPL and JAXA). For the German Earth observation mission proposal Tandem-L he is responsible for SAR system and performance engineering. Currently he is leading the Phase-A and Phase-B1 studies for an L-band mission in the frame of ESA's Copernicus programme. His research interests include digital signal processing, electromagnetic field theory, inverse problems, array processing and antenna theory.

Dr. Huber is the author or co-author of more than 70 publications in international conferences and journals and is holder of a patent on array-fed reflector antennas for synthetic aperture radar applications.

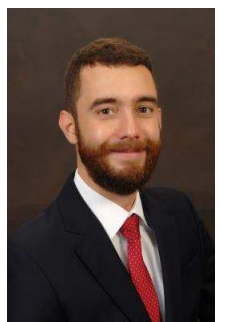

Felipe Queiroz de Almeida received his B.S.E.E. (with honors) and M.S.E.E. degrees in electronic engineering from the Aeronautics Technological Institute (ITA), Sao Jose dos Campos, Brazil, in 2009 and 2011, respectively. He received the Dr.-Ing. (Ph.D.) degree (with honors) in electrical engineering from the Karlsruhe Institute of Technology (KIT), Germany, in 2017.

In 2009, he was a research intern in the Microwaves and Radar Institute of the German Aerospace Center (DLR-HR), where he developed his Bachelor Thesis. From 2011 to 2014, he was with Bradar Industria S.A. in Sao Jose dos Campos, working on software development for Synthetic Aperture Radar applications. Since November 2014, he is again with the DLRHR in Oberpfaffenhofen, Germany, first as a Ph.D. Student and then as a researcher. His research interests include radar signal processing and digital beamforming, in particular beamforming techniques for High-Resolution Wide-Swath SAR.

Dr. Queiroz de Almeida was awarded the EADS Argus Student Award 2010 for his Bachelor Thesis and the Franz-Xaver-Erlacher Award for young scientists in 2015.

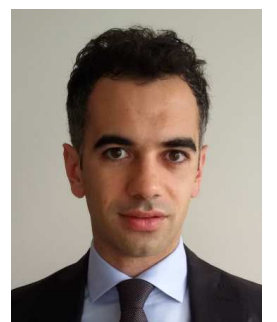

Michelangelo Villano (S'12-M'16) received the B.Sc. and M.Sc. degrees (Hons) in telecommunication engineering from the University of Rome "La Sapienza," Rome, Italy, and the Ph.D. degree (Hons) from the Karlsruhe Institute of Technology, Karlsruhe, Germany, in 2006, 2008, and 2016, respectively.

He was a young graduate trainee with the European Space Research and Technology Center, European Space Agency, Noordwijk, The Netherlands, from September 2008 to August 2009, where he developed processing algorithms for ice sounding radar. Since September 2009, he has been a research scientist with the Microwaves and Radar Institute, German Aerospace Center, Oberpfaffenhofen, Germany, where he works on innovative synthetic aperture radar (SAR) concepts for Earth observation. From April to June 2017 he was a visiting research scientist with the Communications, Tracking, and Radar Division, NASA Jet Propulsion Laboratory, Pasadena, CA, USA, where he analyzed novel acquisition modes for the NASA-ISRO SAR (NISAR) instrument.

He has authored nearly 70 research articles in peer-reviewed journals and international conference proceedings, and he is the holder of two patents in the field of synthetic aperture radar.

Dr. Villano was the recipient of the IEEE Geoscience and Remote Sensing Society Letters Prize Paper Award in 2015 and 2017, the Award as Young Scientist of the Foundation Werner von Siemens Ring 2017, the ITG Dissertation Award 2017, the DLR Science Award 2016, the Student Paper Award at the Asia-Pacific Conference on Synthetic Aperture Radar 2015, Marina Bay Sands, Singapore, and the First Place Student Paper Award at the European Conference on Synthetic Aperture Radar (EUSAR) 2014, Berlin, Germany. He has served as guest editor for the special issues "Advances in Antenna Array Processing for Radar 2014" and "Advances in Antenna Array Processing for Radar 2016" of the International Journal of Antennas and Propagation, and as a member of the Technical Program Committee of the EUSAR Conference since 2016.

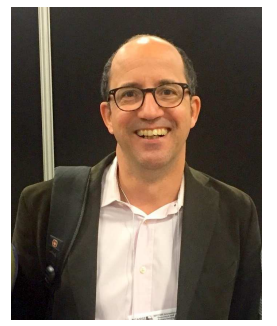

Marwan Younis (S'1995, M'2005, SM'2008) received his B.Sc in electrical engineering from the University of Baghdad, Iraq in 1992 and the Dipl.-Ing. (M.Sc.) and Dr.-Ing. (Ph.D.) degree in electrical engineering from the Universit $\tilde{A}$ at Karlsruhe (TH), Germany, in 1997 and 2004, respectively.

From 1998 to 2004, he was a research scientist with the Institut für Höchstfrequenztechnik und Elektronik, Universität Karlsruhe. Since 2005 he has been with the Microwaves and Radar Institute of the German Aerospace Center (DLR), Oberpfaffenhofen, Germany. He is currently Head of the SAR Techniques Group at the DLR and Professor for Spaceborne Radar Systems at the Karlsruhe Institute for Technology (KIT), Karlsruhe, Germany. He is the author and co-author of about 150 conference papers, and 30 reviewed publications. His research fields include synthetic aperture radar (SAR) systems and techniques, MIMO SAR, digital beamforming, SAR performance, calibration, and antennas. In 1996 he was an intern at the Jet Propulsion Laboratory (JPL) and in 2013 he spent a three month research sabbatical at JPL.

Dr. Younis is an active member of the IEEE and currently director of Corporate Relations for the GRSS. He co-chairs the Instrumentation and Future Technologies GRSS Technical Committee. He is associate editor for the IEEE geoscience and remote sensing letter and reviewer of IEEE publications. He received the Hermann-Billing award for his Ph.D. thesis in 2005. 


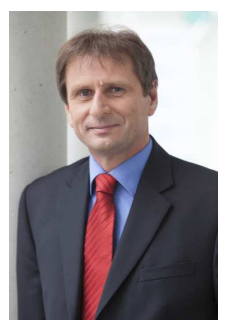

Gerhard Krieger (M'04-SM'09-F'13) received the Dipl.-Ing. (M.S.) and Dr.-Ing. (Ph.D.) (Hons.) degrees in electrical and communication engineering from the Technical University of Munich, Germany, in 1992 and 1999, respectively.

From 1992 to 1999, he was with the Ludwig Maximilians University, Munich, where he conducted multidisciplinary research on neuronal modeling and nonlinear information processing in biological and technical vision systems. Since 1999, he has been with the Microwaves and Radar Institute of the German Aerospace Center (DLR), Oberpfaffenhofen, Germany, where he started as a research associate developing signal processing algorithms for a novel forward-looking radar system employing digital beamforming on receive. From 2001 to 2007, he led the New SAR Missions Group which pioneered the development of advanced bistatic and multistatic radar systems, such as TanDEM-X, as well as innovative multichannel SAR techniques and algorithms for high-resolution wideswath SAR imaging. Since 2008, he has been the Head of the new Radar Concepts Department which hosts about 40 scientists focusing on new SAR techniques, missions and applications. Since 2006, he has also been serving as Mission Engineer for TanDEM-X, and from 2008 to 2016 he led the Phase- 0 and Phase-A studies of Tandem-L, where in the current Phase-B study he is leading the mission and performance engineering. Gerhard Krieger is a Visiting Lecturer with the Friedrich Alexander University, Erlangen, Germany, and he is the author or coauthor of more than 80 peer-reviewed journal papers, 9 invited book chapters, about 400 conference papers, and more than 15 patents.

Dr. Krieger has been an Associate Editor for the IEEE Transactions on Geoscience and Remote Sensing since 2012. In 2014, he served as the Technical Program Chair for the European Conference on Synthetic Aperture Radar and as a Guest Editor for the IEEE Journal of Selected Topics in Applied Earth Observations and Remote Sensing. He received several national and international awards, including the W.R.G. Baker Prize Paper Award from the IEEE Board of Directors and two Transactions Prize Paper Awards of the IEEE Geoscience and Remote Sensing Society.

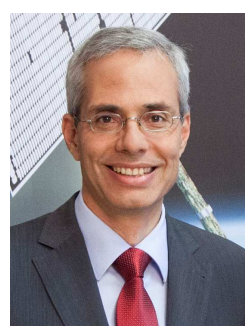

Alberto Moreira (M'92, S'96, F'04) received the B.S.E.E. and M.S.E.E. degrees from the Aeronautical Technological Institute (ITA), Sao Jose dos Campos, in 1984 and 1986, respectively, and the Eng. Dr. degree (with honors) from the Technical University of Munich, Germany, in 1993 . From 1996 to 2001, he was the Chief Scientist and Engineer with the SAR Technology Department, German Aerospace Center (DLR), Oberpfaffenhofen, Germany. Under his leadership, the DLR airborne SAR system has been upgraded to operate in innovative imaging modes like polarimetric SAR interferometry and SAR tomography. Since 2001, Prof. Moreira is the Director of the Microwaves and Radar Institute at DLR and a Full Professor with the Karlsruhe Institute of Technology (KIT), Germany, in the field of microwave remote sensing. His DLR's Institute contributes to several scientific programs and projects for spaceborne SAR missions like TerraSAR-X, TanDEM-X, SAR-Lupe and SAR-Lupe follow-on as well as Kompsat-6, PAZ, Sentinel-1, BIOMASS and Tandem-L. The mission TanDEM-X, led by his Institute, has generated a global, high-resolution digital elevation model of the Earth with unprecedented accuracy. Prof. Moreira is the initiator and Principal Investigator for this mission. His professional interests and research areas encompass spaceborne radar end-to-end system design, microwave techniques and system concepts, signal processing, and remote sensing applications.

Prof. Moreira is author or co-author of more than 350 publications in international conferences and journals, 8 book chapters and is holder of 21 patents in the radar and antenna field. He is an IEEE fellow and has served as President of the IEEE Geoscience and Remote Sensing Society (GRSS) in 2010. He is recipient of several international awards including the IEEE AESS Nathanson Award (1999) for the "Young Radar Engineer of the Year", the IEEE Kiyo Tomiyasu Field Award (2007), IEEE W.R.G. Baker Award from the IEEE Board of Directors (2012), and the IEEE GRSS Distinguished Achievement Award (2014). He was founder and chair of the GRSS German Chapter (2003-2008), served as Associate Editor for the IEEE GRS Letters (2003-2007) and for the IEEE TGRS (since 2005). He and his colleagues received the GRSS Transactions Prize Paper Awards in 1997, 2001, and 2007 and the GRSS Letters Prize Paper Award in 2015 and 2017. Since 2012 he is serving as the Principal Investigator for the Helmholtz Alliance "Remote Sensing and Earth System Dynamics", comprising of 18 research institutes and 30 associated international partners. 